\title{
LARGE-SCALE INTEGRATION OF DNA METHYLATION AND GENE EXPRESSION ARRAY PLATFORMS
}

\author{
A PREPRINT
}

August 12, 2021

Eva E. Lancaster ${ }^{1, *}$, Vladimir I. Vladimirov ${ }^{2}$, Brien P. Riley ${ }^{1,3}$, Joseph W. Landry ${ }^{3}$, Roxann Roberson-Nay ${ }^{1,4}$, Timothy P. York ${ }^{3,5}$

1 Department of Psychiatry, Virginia Commonwealth University, Richmond, VA 23220

2 Department of Psychiatry, Texas A\&M University, College Station, TX, 77843

3 Department of Human and Molecular Genetics, Virginia Commonwealth University, Richmond, VA 23220

4 Department of Psychology, Virginia Commonwealth University, Richmond, VA 23220

5 Department of Obstetrics and Gynecology, Virginia Commonwealth University, Richmond, VA 23220

* Eva.Lancaster@vcuhealth.org

\begin{abstract}
Epigenome-wide association studies (EWAS) aim to provide evidence that marks of DNA methylation (DNAm) have downstream consequences that can result in the development of human diseases. Although these methods have been successful in identifying DNAm patterns associated with disease states, any further characterization of etiologic mechanisms underlying disease remains elusive. This knowledge gap does not originate from a lack of DNAm-trait associations, but rather stems from study design issues that affect the interpretability of EWAS results. Despite known limitations in predicting the function of a particular CpG site, most EWAS maintain the broad assumption that altered DNAm results in a concomitant change of transcription at the most proximal gene. This study integrated DNAm and gene expression (GE) measurements in two cohorts, the Adolescent and Young Adult Twin Study (AYATS) and the Pregnancy, Race, Environment, Genes (PREG) study, to improve the understanding of epigenomic regulatory mechanisms. $\mathrm{CpG}$ sites associated with GE in cis were enriched in areas of transcription factor binding and areas of intermediate-to-low CpG density. CpG sites associated with trans GE were also enriched in areas of known regulatory significance, including enhancer regions. These results highlight issues with restricting DNAm-transcript annotations to small genomic intervals and question the validity of assuming a canonical cis DNAm-GE pathway. Based on these findings, the interpretation of EWAS results is limited in studies without multi-omic support and further research should identify genomic regions in which GE-associated DNAm is overrepresented.
\end{abstract}

\section{Introduction}

Epigenome-wide association studies (EWAS), aiming to test the theory that marks of DNA methylation (DNAm) are involved in the pathophysiology of disease, have successfully identified associations between complex traits and DNAm. ${ }^{11}$ Specific DNAm patterning has been associated with environmental exposures, $\frac{\sqrt[2]{4}}{4}$ as well as short- and long-term health outcomes. ${ }^{5-7]}$ Several attributes of DNAm potentially link this epigenetic mark to the development or progression of complex disease. Appropriate DNAm patterning is essential for normal development and aging, and DNAm regulatory mechanisms are implicated in a multitude of molecular processes, such as cellular differentiation, 
$\mathrm{X}$-inactivation, and genomic imprinting. $\cdot[-11]$ As an epigenetic mark, DNAm is both dynamic and persistent; modifiable by environmental exposures yet heritable during cell division, so that any alterations to DNAm patterns may be carried through future populations of cells. ${ }^{12}[15$ Importantly, altered DNAm has been linked to downstream functional changes, particularly in the regulation of gene expression (GE). These properties suggest that DNAm may be contributing to mechanisms in which previous exposures and genetic predispositions can have lasting effects on disease risk. While EWAS methods are promising, their current utility beyond biomarker discovery is questionable due to study design limitations that impact the interpretability of results, particularly those stemming from the omission of GE measurements. 15

The canonical mechanism describes DNAm as a repressor of proximal transcription, in which DNAm within promoter regions is able to silence GE by either blocking the binding of essential transcriptional machinery or by recruiting chromatin modifying proteins that transition the local DNA conformation to a more heterochromatic state. ${ }^{1016]}$ Despite accumulating evidence that suggests this model is overly simplistic, many researchers rely on this paradigm to interpret an association between DNAm and a disease of interest. In a typical EWAS, any significantly associated CpG sites (also known as differentially methylated positions or DMPs) are each mapped to their most proximal gene and the biological function of those genes is reported in the context of the tested phenotype. Given that this interpretation emphasizes the functional relevance of specific genes to disease biology, an argument can be made that current EWAS are primarily interested in examining a theory of DNAm-driven transcriptional regulation ${ }^{15}$ By inferring transcriptional activity from DNAm-trait associations, this approach relies on assumptions without directly testing for functional evidence. Given that accurately inferring the functional consequences of modified DNAm at any particular site is still very limited, this practice may lead to inaccurate conclusions about disease biology. [17]

Accurately predicting the functional impacts of altered DNAm remains challenging, in part, due to the limited characterization of genome-wide DNAm-GE relationships. 18 DNAm often does not block transcription independently but rather works in concert with other regulatory elements to coordinate GE. These regulatory mechanisms involve a complex crosstalk between DNAm, higher-order chromatin modifiers, and other epigenetic marks, further contributing to difficulties in determining the functional impact from DNAm measurements alone. ${ }^{19}[21]$ Moreover, linking genes to their putative regulatory regions is not always straightforward. ${ }^{[22}$ DMPs are often located outside of proximal regulatory elements, within intergenic or intronic regions with no known regulatory function. Since a frequently utilized approach for interpreting these results involves linking all DMPs to their nearest gene, any features of the genomic landscape beyond distance are disregarded. Even if $\mathrm{CpG}-\mathrm{GE}$ pairs are identified, predicting the regulatory consequence of altered DNAm remains difficult as exceptions to the canonical theory have accumulated. For example, increased DNAm, particularly within the gene body, is frequently positively correlated with local transcription. ${ }^{23+28}$ Although mechanisms linking hypermethylation to increased GE are still unclear, a recent study identified more transcription factors which preferred binding methylated sequences than those inhibited by DNAm. ${ }^{21}$ These functional complexities suggest that assumptions regarding transcriptional activity should not be inferred by DNAm patterns alone. Instead, if the fundamental theory being explored is a mechanism of transcriptional regulation modulated by DNAm, measurements of GE should be included in the analysis. 15

Multi-omic studies integrating global DNAm and GE measurements can provide evidence for DNAm-driven transcriptional regulatory mechanisms. Measuring GE alongside DNAm would allow for direct testing of the proposed mechanism while avoiding assumptions regarding the regulatory function of DNAm that typically cloud the interpretation of EWAS results. In order to model the proposed molecular mechanism, analyses testing DNAm-driven transcriptional regulation should provide evidence for the mediating effect of GE on the phenotype of interest. Instead, studies often discover differentially methylated and differentially expressed genes separately by performing both an EWAS and transcriptome-wide association study, and any overlaps between DNAm-trait and GE-trait associations are reported. ${ }^{29}[36]$ While the addition of GE-trait associations provides more support for functional changes within the cell, it is unclear what hypothesized biological mechanism this analysis is testing. Moreover, since the relationship between DNAm and GE is never tested, this method still relies on assumptions that DMPs strictly influence expression of the nearest transcript.

An extended EWAS approach integrating both DNAm and GE measurements holds promise in uncovering biological processes important to the development or progression of disease, however, a mechanistic interpretation requires prior knowledge regarding specific DNAm-GE relationships across the genome, which has yet to be resolved. The objective of this study was to catalogue the relationships between DNAm and both proximal and distal GE (i.e., cis and trans relationships, respectively) in peripheral blood, a tissue commonly assayed in EWAS. To identify attributes that replicate across disparate samples, analyses were conducted in two previously described cohorts, the Adolescent and Young Adult Twin Study (AYATS), 737 and the Pregnancy, Race, Environment, Genes Study (PREG) ${ }^{38}$ An in-depth characterization of GE-associated $\mathrm{CpG}$ sites could improve predictions of the downstream functional impact of altered DNAm and inform best practices for interpreting DNAm-trait associations generated by EWAS. 
A PREPRINT - AUgust 12, 2021

\section{Methods}

\subsection{Study cohorts}

Adolescent and Young Adult Twin Study (AYATS). The AYATS study was designed to examine genetic and environmental contributions to internalizing pathways (e.g., depression and anxiety) during development. A sample of monozygotic twins were chosen for their adherence to the study's inclusion criteria (e.g., 15-20 years of age, no current use of psychotropic medications). ${ }^{7377}$ Peripheral blood collected from 141 participants at a single time point was assayed for both DNAm and GE. An overview of study characteristics and further demographic information can be accessed in the supplement (Supplementary Table S1).

Pregnancy, Race, Environment, Genes (PREG) study. The PREG Study is a prospective longitudinal study with the purpose of identifying how environmental determinants of health and DNAm remodeling relate to racial health disparities in perinatal health outcomes. $\frac{38}{38}$ Of the 240 women who enrolled in the study, 177 met all birth and pregnancy inclusion criteria (e.g., mother and father self-identify as either both Caucasian or both African American) and no exclusion criteria (e.g., preeclampsia, fetal congenital anomaly, placental anomaly, fewer than 3 study time points completed). Peripheral blood samples were collected up to four times throughout pregnancy. Sample collection was scheduled during gestational weeks $0-15,10-25,20-40$, and 37-42. DNAm was assessed at all time points whereas GE was measured once at the final collection during weeks 37-42. Only those DNAm measurements from specimen simultaneously collected with GE were analyzed in this study. A total of 151 women had concomitant DNAm and GE measured. An overview of study characteristics and further demographic information can be found in the supplement (Supplementary Table S2).

\subsection{DNAm measurement and data processing}

In both samples, DNAm and GE was measured from peripheral blood. The Infinium 450k HumanMethylation BeadChip assayed genome-wide DNAm and the Affymetrix HG-U133A 2.0 array measured GE. A description of platform characteristics as well as the methods used for measurement and preprocessing can be found in the supplement.

\subsection{Association analysis}

The relationship between all pairwise combinations of measured DNAm and GE (Table 1) was tested by linear regression in the R statistical environment (version 3.5). ${ }^{39}$ Log-transformed expression values (dependent variable) were regressed on DNAm M-values (independent variable), while covariates controlled for differences in cell type heterogeneity. Cell type proportions were derived from the Houseman algorithm, which estimates proportions for granulocytes, monocytes, CD8-positive T cells, CD4-positive T cells, B lymphocytes, and natural killer cells based on cell type-specific DNAm profiles. ${ }^{[0]}$ Granulocytes were selected to account for overall differences in cell type proportions based on high correlations with other cell type estimates (absolute correlations ranged from 0.47 to 0.71 ), and included as a covariate in all models. Natural killer proportions were included in AYATS models exclusively to adjust for the atypical variation in this cellular fraction characteristic of depressed patients.

Additional covariates were selected to adjust for potential confounding influences specific to the characteristics of each cohort, while also maintaining a similar analytical approach across the two studies. Since PREG is a racially diverse sample (Table 1), ancestrally informative principal components were estimated from the DNAm data using the method described in Barfield et al. The third principal component was highly correlated with self-reported race and included as a covariate in the PREG cohort models. ${ }^{42}$ A linear mixed-model framework was used to account for twin structure in the AYATS cohort. ${ }^{43}$ The limma Bioconductor package was used to estimate within-family correlations from 1,000 randomly sampled $\mathrm{CpGs}$ in order to appropriately adjust model standard errors and account for the non-independence of twin pair DNAm observations. ${ }^{43}$

Both DNAm and GE measurements were adjusted for technical artifacts prior to analysis (see supplement), so that variables related to slide or row effects were not included as covariates in subsequent analyses.

Although measurements were generated using the same technology in both cohorts, differing numbers of probes remained after quality control procedures (Table 1). A within-study Bonferroni correction was used to adjust for multiple testing at an alpha threshold of 0.05 . While estimates of genomic inflation are typically used to identify spurious associations driven by artefacts in genome-wide association studies (GWAS), it has been recently suggested that inflated test statistics should be similarly reviewed in epigenetic studies. ${ }^{44}$ To mitigate the presence of false positives, genomic inflation was assessed using the method described in Kennedy et al. ${ }^{24}$ Briefly, genomic inflation factors were calculated for each transcript, across all $\mathrm{CpG}$ associations, as the median (T-statistic) ${ }^{2} / 0.4549$. Appropriate thresholds 
for test statistic inflation are not as well established in the epigenetics field. To facilitate cross-study comparisons, any transcript with an inflation factor $>2$ was flagged for removal. ${ }^{[2]}$

Every pairwise relationship between measured DNAm and GE was modeled and classified as either cis or trans, since molecular mechanisms linking proximal DNAm may differ from more long-range interactions. DNAm-GE pairs were in cis if the $\mathrm{CpG}$ site was located within a gene or 2,500 base pairs upstream. This extension is expected to capture important transcript-specific regulatory regions, given that many promoters are located up to 1 kilobase upstream of the transcriptional start site (TSS) ${ }^{45} \mathrm{CpG}$-transcript pairs located outside this range were categorized as trans relationships, with the rationale that more distal regulatory features (e.g., enhancers) may be responsible for the relationship between $\mathrm{CpG}$ methylation and transcript expression.

\subsection{Characterization of results}

CpG sites were mapped to biologically relevant annotations to test for feature enrichment among sites significantly associated with GE. Annotation selection was based on evidence that local $\mathrm{CpG}$ density, gene feature location, and proximity to regulatory elements are important characteristics that may impact the functional consequences of DNAm. 16 Transcription factor activity is regulated by DNAm, and a history of transcription factor binding also appears to influence the susceptibility of $\mathrm{CpG}$ methylation in specific locations. $\frac{2146}{2148}$ Moreover, processes involving transcription factors were previously enriched among CpGs associated with GE. ${ }^{2449}$ Similarly, non-coding RNAs are regulated by methylation patterning, while also contributing to the regulatory activity of DNAm. 50 [51 Chromatin states are accurately able to distinguish variable transcriptional activity by describing the specific patterns of histone modifications that impact the regulation of GE. ${ }^{5253}$ Histone modifications are intricately linked to both DNAm and GE, potentially serving to mediate the influence of methylation on transcription. 1652

Selected features described local CpG densities (UCSC CpG island classifiers and HIL annotations), ${ }^{5455}$ genomic regions, chromatin states (ENCODE 15-state ChromHMM), ${ }^{53}$ transcription factor binding (ENCODE TF ChIP-seq), 56 non-coding RNAs (GENCODE version 37), ${ }^{[57]}$ and other annotations related to regulatory activity (i.e., FANTOM5defined enhancer and ENCODE-defined insulator regions). ${ }^{58}$ All cell type-specific annotations (e.g., chromatin states, enhancer regions, etc.) were defined in the lymphoblastoid cell line GM12878.

Enrichment analyses were performed separately for cis and trans groups. The proportion of significant findings annotated to each category was compared to the proportion of total number of tested $\mathrm{CpG}$ sites using Fisher's exact test. A Bonferroni correction for 20 enrichment tests was used to adjust for unique annotation categories examined (e.g., CpG density classifiers, chromatin states, transcription factor binding, etc.)

\section{Results}

\subsection{Participant demographics and initial findings}

After performing the preprocessing procedures described in the supplementary methods, all 137 of the remaining GE measurements also had corresponding DNAm of sufficient quality in AYATS. In PREG, 131 samples had both DNAm and GE that passed quality control. While the tissue and platforms were consistent across studies, these cohorts differed in other characteristics (Table 11). PREG was an older (aged 18-40 years) and more racially diverse sample, with $49 \%$ of participants reporting African American ancestry (Supplementary Table S2). Notably, all participants in the PREG sample were pregnant women, while the AYATS sample consisted of both male (29\%) and female (71\%) adolescents (aged 15-20 years; Supplementary Table S1).

Genome-wide methylomic and transcriptomic data was generated using the Illumina HumanMethylation 450k BeadChip and Affymetrix HG-U133A 2.0 array, respectively. After performing quality control procedures separately in both cohorts, a differing number of probes were identified as poor quality. In AYATS, 40,392 DNAm probes and 10,809 GE probe sets were removed during preprocessing, while 63,783 DNAm and 9,473 GE measurements were removed in PREG (Table 1 .

\subsection{Associations between DNA methylation and gene expression}

\subsubsection{Overall findings}

An overview of significant DNAm-GE relationships is presented in Table 2 (see the Open Science Framework project page at https://osf.io/dk3cg/for full lists of associations with summary statistics). Due to the differing number of measurements surviving quality control, associations with p-values $<9.68 \times 10^{-12}(0.05$ alpha corrected for $5,165,758,521$ total tests) in PREG and p-values $<1.03 \times 10^{-11}$ ( 0.05 alpha corrected for 4,857,594,560 tests) in 
AYATS were considered significant (Table 2). A total of 903 associations were identified in the AYATS cohort, 169 of which were in cis $\left(4.72 \times 10^{-61}<\mathrm{p}<1.01 \times 10^{-11}\right)$ and 734 in trans $\left(2.64 \times 10^{-61}<\mathrm{p}<1.03 \times 10^{-11}\right)$. Within the PREG sample, 379 DNAm-GE associations were statistically significant, of which 121 were cis $\left(5.15 \times 10^{-58}\right.$ $\left.<\mathrm{p}<8.50 \times 10^{-12}\right)$ and 258 trans $\left(2.86 \times 10^{-53}<\mathrm{p}<9.51 \times 10^{-12}\right)$. Since GE probe sets measuring expression of the same gene were retained, some transcripts and $\mathrm{CpG}$ sites are represented more than once in the results. A total of 340 unique CpG sites and 105 unique genes comprised the 903 significant associations identified in AYATS, while 228 CpGs and 69 genes were unique in PREG across both cis and trans relationships (total $\mathrm{n}=379$ ). Across all categories (i.e., AYATS/PREG cis/trans), many significant relationships occurred between one transcript and one CpG site (Supplementary Figures S1 and S2), although instances in which a single CpG site was associated with multiple transcripts, and vice versa, were also common. Both positive and negative relationships were identified, although the majority of significant associations had negative coefficients (49\% to 78\% negative across tested categories; Table 2 and Figure 11). Effect sizes were relatively large throughout (adjusted R-squared range $=0.23-0.90$ ), with $\mathrm{cis}$ DNAm explaining more GE variability on average (mean adjusted R-squared $=0.58$ and 0.46 for AYATS and PREG, respectively) when compared to trans (mean adjusted R-squared $=0.48$ and 0.40 for AYATS and PREG, respectively).

AYATS Associations. The distribution of significant cis and trans connections is shown in Figure $2 \mathrm{a}$ On average, each significant cis $\mathrm{CpG}$ site was associated with 1.58 transcripts (median =1, range =1-4; Supplementary Figure S1). Trans $\mathrm{CpGs}$ were more likely to associate with multiple transcripts than cis (mean $=2.75$, median $=1$, range $=1-22$ ). Effect sizes, defined by adjusted R-squared values, ranged from 0.23 to 0.90. Cis DNAm explained more variation in GE on average (Welch's t-test $\left.\mathrm{p}=2.2 \times 10^{-16}\right)$. DNAm-GE relationships were predominantly negative $(65 \%$ of cis and $78 \%$ of trans relationships; Table 2 and Figure 1 .

PREG Associations. The distribution of significant cis and trans connections is shown on Figure $2 \mathrm{~b}$. On average, each significant cis $\mathrm{CpG}$ site associated with 1.39 transcripts (median = 1, range =1-4; Supplementary Figure S1). Significant trans CpGs were more likely to associate with multiple transcripts (mean $=1.65$, median $=1$, range $=1-11$ ). Effect sizes ranged from 0.31 to 0.86 , with cis DNAm explaining more variation in GE on average (Welch's t-test $\mathrm{p}=$ $\left.5.10 \times 10^{-08}\right)$. Cis DNAm-GE relationships were predominantly negative (79\%) while trans relationships were split almost equally between positive and negative associations (Table 22.

\subsubsection{Between study comparison}

A total of 86 individual DNAm-transcript pairs replicated across cohorts (57 cis and 29 trans). In cis, $34 \%$ of significant CpG-GE pairs identified in AYATS were replicated, and 47\% of those found in PREG overlapped with AYATS. In trans, only $4 \%$ of AYATS and $11 \%$ of PREG connections were replicated.

\subsubsection{Location relative to transcriptional start sites}

Previous research has suggested that DNAm adjacent to the gene TSS has a stronger role in regulating proximal GE. ${ }^{24 \mid 4959}$ To explore this topic further, CpG sites associated with GE in cis were mapped to their associated TSS, as defined by annotations from the UCSC hg 19 build knownGene track. ${ }^{60}$ The location of cis CpG sites relative to the TSS of their associated gene is shown in Figure 3 . Although many significant sites were located near the 5' end of gene boundaries, these areas are also overrepresented in the 450k microarray. Overall, the relative proportion ([number GE-associated CpGs within 2500bp / total number GE-associated CpGs] / [number microarray CpGs within 2500bp / total number microarray $\mathrm{CpGs}]$ ) of $\mathrm{CpGs}$ was higher in the GE-associated $\mathrm{CpG}$ sites compared to the microarray background. Interestingly, this observation was driven by $\mathrm{CpG}$ sites located downstream of the TSS. The relative proportion of CpG sites 2500bp upstream of the TSS was lower in GE-associated CpGs than was present on the microarray, while the opposite relationship was observed immediately downstream of the TSS (relative proportion upstream $=0.58$ and 0.65 ; relative proportion downstream $=1.59$ and 2.21 in AYATS and PREG, respectively).

\subsection{Enrichment analyses}

CpG sites were annotated by genomic regions, local $\mathrm{CpG}$ densities, chromatin states, bound transcription factors, and other related regulatory regions (e.g., insulator regions, regulatory RNAs). Enrichment tests were then performed within annotation type. An overview of results from enrichment analyses is outlined in Tables 3 (AYATS) and 4 (PREG). Annotation categories with p-values $<0.0025$ exhibited significant depletion or enrichment, while $\mathrm{p}$-values $<0.05$ were considered suggestive. A number of depleted and enriched categories overlapped between the two cohorts (underlined in Tables 3 and 4. Overall, regions of high CpG density were depleted across all groups (Supplementary Table S3 and Supplementary Figures S3 - S4) while annotations indicative of regulatory activity (e.g., transcription factor binding, enhancers) were enriched among GE-associated CpGs (Tables 3- 4). 
A PREPRINT - AUgust 12, 2021

\subsubsection{Characterization of cis connections}

South shore regions (i.e., shore regions located downstream from a $\mathrm{CpG}$ island) were significantly $(\mathrm{p}<0.0025)$ or suggestively $(\mathrm{p}<0.05)$ enriched across all groups (Figure 4). $\mathrm{CpG}$ islands are often associated with promoters, and both of these annotations were depleted in AYATS but were neither significantly enriched or depleted in PREG (Figures 4 and 5). Transcription factor binding sites, defined by significant peaks identified in ChIP-seq analyses of 134 transcription factors in lymphoblastoid cells,, 56 were enriched in both cohorts (Figure 6). Chromatin state characteristics, which assign a function to genomic regions based on the presence of specific histone methylation marks, also showed some concordance between the two studies (Figure 77). Specifically, zinc-finger genes and repeats were consistently found to be enriched, whereas areas of strong transcription were consistently depleted. Regions flanking active transcription were more variably assigned, with one category found to be enriched in AYATS (areas flanking strong transcription) and another depleted in PREG (areas flanking active TSS).

\subsubsection{Characterization of trans connections}

Like cis CpG-GE pairings, CpGs associated with GE in trans were overall depleted in areas of high CpG density (i.e., within $\mathrm{CpG}$ islands) and within the promoter regions of genes, while South shore regions were enriched (Tables 3 - 4 and Figures 8- 9). Again, GE-associated $\mathrm{CpG}$ sites were overrepresented in areas of known regulatory importance, such as sites of transcription factor binding and enhancer regions (Figure 10). With the exception of IncRNAs, which were depleted in AYATS, noncoding RNAs were neither over- or underrepresented. The chromatin state analysis highlighted distinct differences between cis and trans results. Chromatin states reflecting enhancer regions were enriched in both AYATS and PREG, as were areas flanking sites of active transcription. Repressed states, including heterochromatic regions and polycomb-repressed regions, were consistently depleted (Figure 11).

\subsubsection{Functional enrichment analysis}

The Gene Ontology (GO) Consortium and the Kyoto Encyclopedia of Genes and Genomes (KEGG) were used to assess overrepresented gene functions and pathways within significant $c i$ results (see supplement for more information). Terms with a false discovery rate (FDR) $<0.05$ were deemed significant. ${ }^{61}$ Common themes were uncovered in both cohorts (Supplementary Tables S4 - S11), and include functions related to the activation and regulation of immune response and cellular detoxification. A total of 33 significantly enriched GO terms overlapped between the two cohorts, and all significant KEGG pathways identified in AYATS $(n=31)$ were also found in PREG $(n=39)$. However, these consistences were supported by relatively few genes.

\section{Discussion}

Although genome-wide epigenetic studies aim to uncover the role of DNAm in disease development and progression, they often do not utilize an experimental framework that provides evidence for a mechanistic relationship. Most EWAS operate under the assumption that DNAm influences proximal gene transcription. However, the absence of measured GE makes relying on this interpretation difficult, especially as mounting evidence suggests that DNAm does not always follow a canonical cis relationship. $\frac{[1516]}{16}$ Given the complicated network of interactions between DNAm, GE, higher-order chromatin modifiers, and other regulatory elements, it is challenging to draw accurate conclusions about the downstream functional effects of altered DNAm without, at minimum, integrating concomitant measurements of GE. $\frac{15 / 16 / 20156}{}$ To investigate the relationship between DNAm and GE further, this study tested genome-wide associations between DNAm and GE in peripheral blood collected from two cohorts. Both canonical and non-canonical relationships were identified, highlighting potential inaccuracies in the current functional interpretation of trait-associated $\mathrm{CpG}$ sites within the frequently adopted EWAS framework.

Across cohorts, DNAm was significantly associated with both proximal (cis) and distal (trans) GE. The primary findings of this study align with other reports of long-range DNAm-GE relationships, adding to the growing body of literature questioning the accuracy of current EWAS interpretations. $\frac{1724 / 62}{24}$ The effect sizes detected among DNAm-GE pairs were relatively large, with DNAm predicting $42-50 \%$ percent of GE variability on average. Although this result is likely influenced by a lack of statistical power to detect more attenuated relationships, it reiterates that while DNAm may not be an appropriate proxy for changes in GE, strong links between the two measurements exist. Approximately $23 \%$ of connections identified in PREG were also significant in the AYATS cohort, suggesting a consistent program of gene regulation even among the disparate cohorts tested. While this proportion is similar to DNAm-GE connections identified in peripheral blood and isolated monocytes, ${ }^{[2]}$ discrepancies between the two cohorts could be related to differences in statistical power or differences in demographic and clinical features (i.e., genetic ancestry, developmental stage, etc.). On average, cis connections were more likely to replicate between studies and account for larger proportion 
of GE variability when compared to within-cohort trans associations. Larger samples are likely necessary to detect more subtle cis and trans CpG-GE pairings and provide a balanced assessment of the expected replication across samples.

Interpreting DNAm-disease relationships is hindered not only by limitations in identifying DNAm-GE pairs, but also by challenges in predicting the precise functional impact of altered DNAm on an associated gene's expression. Both negative and positive relationships between cis and trans DNAm-GE pairs were identified (Figure 1). Although DNAm is usually considered a repressive mark, inhibiting GE by either blocking transcription factor binding or by promoting a more condensed DNA conformation, 16 positive DNAm-GE relationships could be explained by several mechanisms. Within genomic regulatory elements, transcription factors with repressive, rather than activating properties, may bind unmethylated sequences. ${ }^{23}$ Furthermore, many transcription factors actually exhibit an increased affinity for heavily methylated sites. ${ }^{21}$ Besides influencing the binding affinity of regulatory proteins, DNAm patterns may also reflect a history of transcription factor binding, a phenomenon that cannot be separately identified by a classic EWAS design. 63 In recent years, speculation has emerged regarding potential alternative roles of DNAm in the cell, including theories that DNAm may serve to direct splicing regulation or in maintaining genomic stability within specific regions. $5[64] 67$ Although this study found that the associations were predominantly negative across the majority of gene regions (Figure 11, these findings agree with other reports that strong positive DNAm-GE relationships exist. 24/25/49

Given the large effect size distribution of detected associations and the modest number of participants in each cohort, it was expected that only a small proportion of DNAm-GE connections would reach statistical significance. Instead of only focusing on individual connections, this study sought to outline genome-wide trends by identifying attributes of GE-associated CpG sites. Despite the modest number of DNAm-GE pairs overlapping across cohorts, GE-associated CpG sites displayed similar annotation characteristics (Tables 3 and 4). Annotations uniquely characterized attributes of cis and trans GE-associated CpGs, indicating that separate paradigms may exist for proximal and distal connections. In general, DNAm within intermediate $\mathrm{CpG}$ density regions were more likely to be associated with GE. Regions of intermediate CG density are more variable compared with low- or high-density regions, and appear more dynamic across tissues and developmental stages. $\frac{6869}{}$ Conversely, CpG sites in high density regions, which are most often associated with $\mathrm{CpG}$ islands and promoter regions, were consistently depleted. Interestingly, both transcription factor binding and chromatin states in regions near active TSS were enriched, with those regions directly downstream of the TSS particularly characterized by a high proportion of GE-associated CpGs (Figure 3). Other studies have noted a similar relationship with DNAm located in the first intron, while also observing high transcription factor activity typical of intronic enhancers within these areas. $\frac{597071}{1}$ Although mechanisms of DNAm transcriptional inactivation usually focus on the hypermethylation of $\mathrm{CpG}$ sites within promoter and island regions, these results agree with other studies showing enrichment for "off-island" DNAm among GE-associated CpG sites. $24 \mid 68$

The traditional DNAm regulatory paradigm suggests the importance of DNAm in promoter regions, but results from this study instead reiterate the significance of DNAm within enhancer regions. ${ }^{24 \mid 27}$ Multiple enhancer definitions (i.e., enhancer-like chromatin states and enhancer annotations generated from cap analysis of gene expression [CAGE]) were enriched within trans results. Enhancers have an established role in long-range gene regulation, ${ }^{[972}$ often looping over more proximal genes to interact with those farther away. ${ }^{[3]}$ Enhancer regions are often characterized by intermediate DNAm and chromatin accessibility, demonstrate greater DNAm variability than promoters, ${ }^{5268}$ and exhibit ongoing de novo methylation and demethylation activity. ${ }^{48}$ The high rate of DNAm remodeling within enhancers, coupled with the strong DNAm-GE relationships found within these regions, align with hypotheses that suggest environmental exposures can influence complex disease risk through epigenetic mechanisms of transcriptional dysregulation. While mapping enhancers to their putative genes is a fundamental aim in identifying transcriptional regulatory networks, current methods are still under development, $\frac{73}{}$ adding to the uncertainty in predicting the downstream functional effects of DNAm within these distal regulatory regions. Further challenges arise from evidence that many genes actually interact with multiple enhancers, and that these compounded interactions can result in additive effects on target GE. ${ }^{73}$ However, the relationships between DNAm outside of proximal regulatory elements and GE again question the generalizability of the canonical DNAm regulatory mechanism and suggests that EWAS should transition away from relying on this paradigm to interpret underlying disease biology.

This study serves to improve understanding of the relationships between DNAm and GE across the genome and adds to the growing body of literature which cautions against misconstruing modified DNAm as changes in proximal GE. Overall, these results highlight issues with restricting DNAm-transcript annotations to small genomic intervals and question the validity of assuming a canonical cis DNAm-GE pathway when investigating epigenetic mechanisms. The results from this study underscore concerns in predicting the biological mechanisms underlying disease from DNAm measurements alone. EWAS relying on a canonical DNAm-mediated transcriptional regulatory mechanism to interpret DNAm-trait associations may reach inaccurate conclusions about disease pathoetiology. Even modified EWAS that incorporate GE information by performing a transcriptome-wide association study alongside testing for DNAm-disease associations should interpret findings with care, since in this study design a priori assumptions link CpG sites to putative genes and DNAm-GE relationships remain uninvestigated. 
Based on these results, epigenetic research should continue moving towards multi-omic approaches that integrate DNAm with other levels of data (e.g., GE, genotypes, transcription factor binding) to study complex traits. Although DNAmGE relationships are highly complex, the integration of DNAm with data outlining regional chromatin architecture and transcription factor activity may assist in predicting the functional impact of altered DNAm. ${ }^{2474}$ However, as an emerging and heterogeneous field, several obstacles can interfere with the implementation and interpretation of multi-omic studies. ${ }^{75}$-78 Standardized analytical pipelines have yet to be developed, leading to difficulties in cross-study comparisons and in assessing rigor. ${ }^{77}$ Currently, only a handful of studies have tested genome-wide associations between GE and DNAm, $24[6268 / 7980]$ but variability in the methodologies used has lead to difficulties in determining the replicability and generalizability of identified relationships. Although cross-study comparisons are challenging, several consistent themes have emerged from this modest body of literature. This study replicates the overrepresentation of GE-associated CpGs within enhancers and at transcription factor binding sites, as well as the depletion within islands and promoter regions. ${ }^{[24}$ Moving forward, continued examination of DNAm-GE relationships in large, diverse cohorts should be prioritized to advance our understanding of the role of DNAm within the cell and disease biology.

\section{Strengths and Limitations}

To our knowledge, this was the first study to assess the global relationship between peripheral blood DNAm and GE in both a primary and replication sample. However, results of this study should be considered in the context of the following limitations. First, both DNAm and GE were measured by microarray technologies that provided coverage within well-characterized locations, but were unable to assay the full extent of RNA and CpG sites in the genome. ${ }^{81}$ Second, only relationships of relatively large effect size were detected in this study (adjusted R-squared range $=0.23-0.90)$. Especially given that a conservative multiple testing correction was applied, it is assumed that many more DNAm-GE connections exist but were undetected in this study, which could influence the results of the feature enrichment tests. Third, both cohorts were analyzed cross-sectionally, a study design that is unable to provide evidence for causation or directionality. ${ }^{[1 / 82}$ Mechanisms of reverse causation, in which changes to DNAm occur in response to modified GE, have been observed. $\frac{83}{3}$ Therefore, it is unknown whether changes to DNAm are actually proceeding changes in GE as described in the canonical mechanism. Fourth, some annotations were derived from experiments conducted on a well-described lymphoblastoid cell line (GM12787), which was selected based on data that supports the genetic and functional similarity to mature blood cells (i.e., T cells and B cells). ${ }^{84}$ One benefit of using this approach is that annotations were kept consistent across the different functional enrichment categories (e.g., chromatin landscapes, enhancer definitions, etc.). It remains important to consider that this study focused on the association between DNAm at individual $\mathrm{CpG}$ sites and GE. In actuality, regional changes in DNAm may also be co-regulating GE together. ${ }^{62[85]}$ While correlations between proximal GE-associated $\mathrm{CpG}$ sites did not suggest a predictable method in which to aggregate measured sites, future studies can examine how regions of $\mathrm{CpGs}$ work in concert to regulate GE 62 Finally, this study only investigated DNAm and GE in the peripheral blood and may not generalize to other tissues. ${ }^{59}$ Future analyses with more comprehensive measurements in alternative tissues will be crucial for characterizing genome-wide trends across cell types. 
A PREPRINT - AUgust 12, 2021

\section{Additional information}

\subsection{Acknowledgements}

The PREG study was supported by the NIMHD (P60MD002256, PI: York) and by the Clinical and Translational Science Award (CTSA) award No. UL1TR000058 from the National Center for Advancing Translational Sciences. Support for the AYATS study was received by a NIH/NIMH R01 (MH101518), R21 (MH106924), and a NARSAD Independent Investigator Award from the Brain and Behavior Research Foundation (PI: Roberson-Nay).

\subsection{Data sharing}

Sharing PREG and AYATS study data is limited by Institutional Review Board agreements and participant consent forms, which restrict openly sharing individual-level measures. Anyone interested in data access or collaboration is encouraged to contact Dr. Timothy P. York (timothy.york@vcuhealth.org) or Dr. Roxann Roberson-Nay (roxann.robersonnay@vcuhealth.org) for more information.

\subsection{Author contributions}

All authors assisted with the design of the study. EL cleaned the data, performed the analyses, and wrote the initial draft of the manuscript. RRN, VV, BR, JL and TY provided substantive feedback and revisions, and TY and RRN planned and secured funding for the PREG and AYATS studies.

\subsection{Conflicts of interest}

The authors report that they have no conflicts of interest to declare.

\subsection{Abbreviations}

AYATS Adolescent and Young Adult Twin Study

DMPs Differentially methylated positions

DNAm DNA methylation

EWAS Epigenome-wide association study

FDR False discovery rate

GE Gene expression

GO Gene ontology

KEGG Kyoto Encyclopedia of Genes and Genomes

PREG Pregnancy, Race, Environment, Genes study

TSS Transcriptional start site

UTRs Untranslated regions 
A PREPRINT - AUGUST 12, 2021

\section{$7 \quad$ Tables and Figures}

Table 1: Study Characteristics

\begin{tabular}{|c|c|c|}
\hline & AYATS & PREG \\
\hline $\mathrm{N}$ & 137 & 131 \\
\hline Study Phenotype & $\begin{array}{l}\text { Internalizing disorders } \\
\text { (e.g., early-onset major depression) }\end{array}$ & $\begin{array}{l}\text { Perinatal health outcomes } \\
\text { (e.g., preterm birth) }\end{array}$ \\
\hline Age & $16.96(1.28)$ & $29.06(4.99)$ \\
\hline Sex (\% female $)$ & $97(71 \%)$ & $131(100 \%)$ \\
\hline Ethnicity (\% Caucasian) & $132(97 \%)$ & $67(51 \%)$ \\
\hline Methylation Probes Tested & 445120 & 421729 \\
\hline Expression Probes Tested & 10913 & 12249 \\
\hline
\end{tabular}

Study characteristics were assessed after preprocessing and removal of poor quality samples. Mean (standard deviation) or $\mathrm{N}(\%)$

Abbreviations. AYATS $=$ Adolescent and Young Adult Twin Study, PREG = Pregnancy, Race, Environment, Genes cohort. 
Table 2: Overview of Pairwise DNAm-GE Association Results

\begin{tabular}{clllll}
\hline Study & $\begin{array}{c}\text { Significant } \\
\text { associations }\end{array}$ & $\begin{array}{c}\text { Direction of } \\
\text { relationship }\end{array}$ & Unique CpGs & Unique transcripts & \begin{tabular}{c} 
Mean adjusted $^{\text {R-squared }}{ }^{5}$ \\
\hline AYATS $^{2}$
\end{tabular} \\
$\quad$ cis & 169 & $65 \%$ negative & 107 & 42 & \\
$\quad$ trans & 734 & $78 \%$ negative & 266 & 96 & $0.58(0.11)$ \\
PREG $^{3}$ & & & & & $0.48(0.10)$ \\
cis & 121 & $79 \%$ negative & 87 & 31 & $0.46(0.10)$ \\
trans & 258 & $49 \%$ negative & 156 & 43 & $0.40(0.08)$ \\
\hline
\end{tabular}

${ }^{1}$ After Bonferroni adjustment for total number of tests performed within cohort

${ }^{2} P$-val $<1.03 \times 10^{-11}$

${ }^{3} P$-val $<9.68 \times 10^{-12}$

${ }_{5}^{4}$ Defined as unique by Entrez identifier

${ }^{5}$ Mean (standard deviation)

Abbreviations. AYATS = Adolescent and Young Adult Twin Study, PREG = Pregnancy, Race, Environment, Genes cohort. 


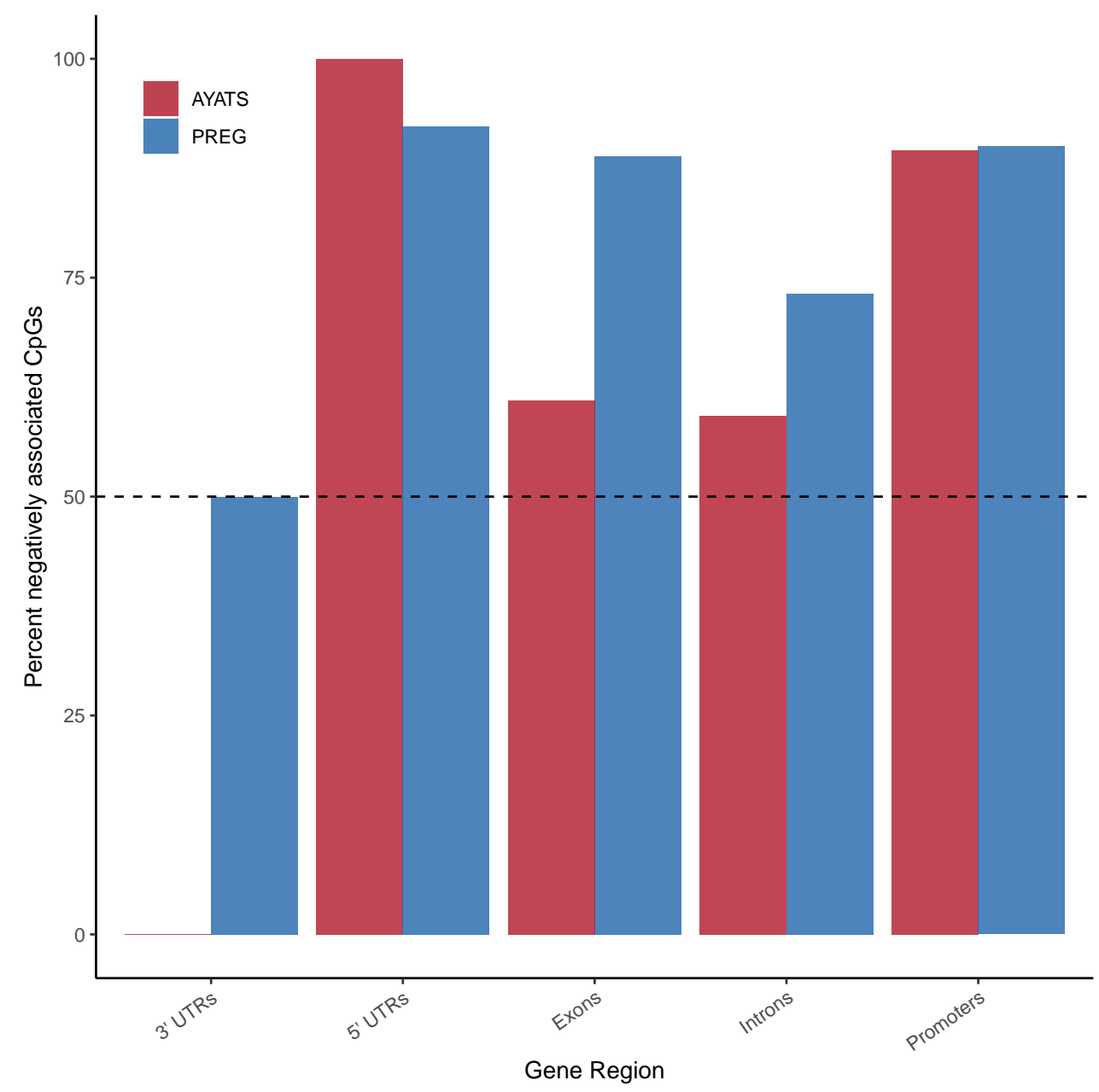

Figure 1: Percent negative CpG-GE associations by gene region. With the exception of 3' untranslated regions (UTRs), the majority of cis CpG-GE relationships were negative across gene regions in both the AYATS (red) and PREG (blue) cohorts. Promoters and 5' UTRs had the highest fraction of negative associations, aligning with canonical descriptions of promoter DNAm as a repressor of local gene transcription. 


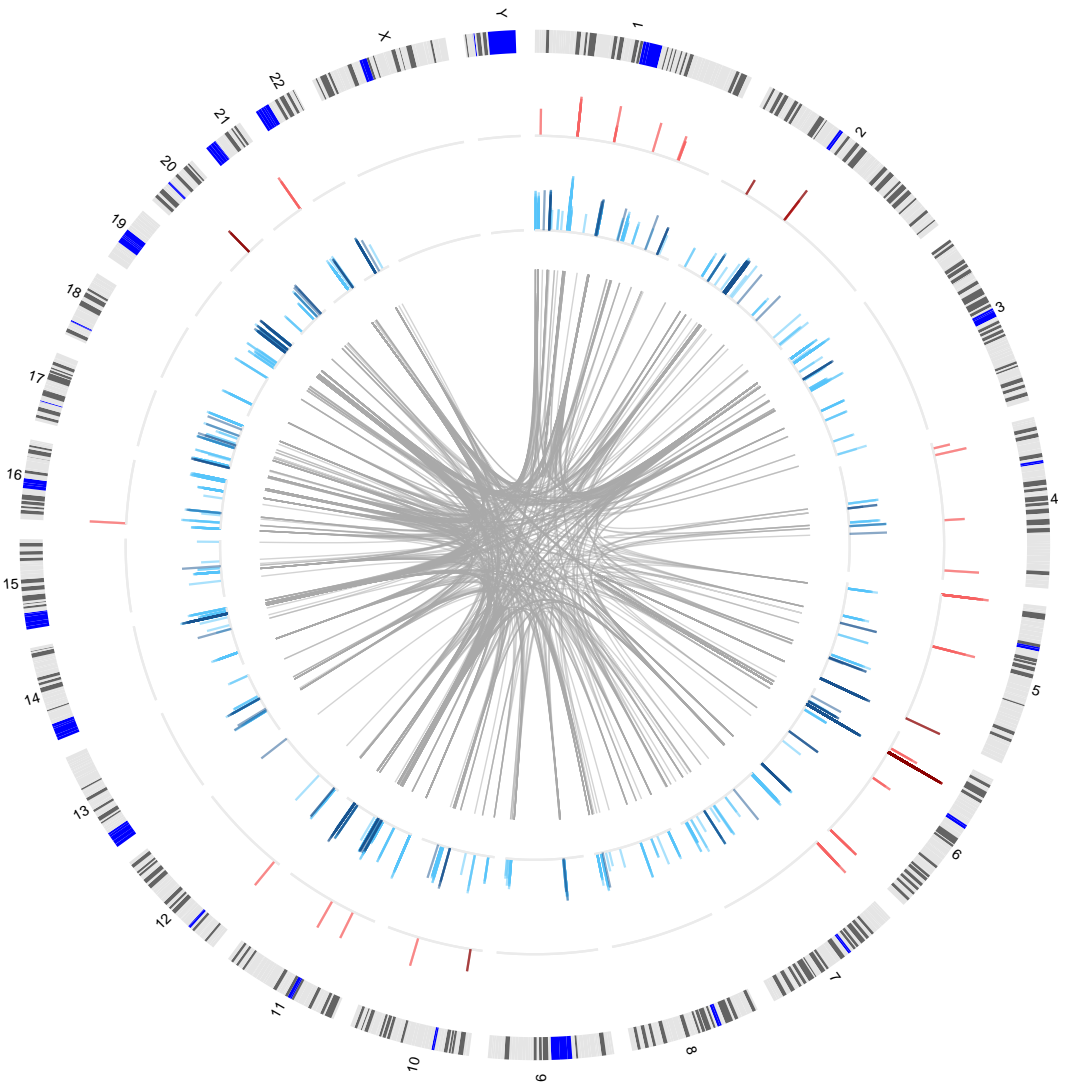

(a) AYATS

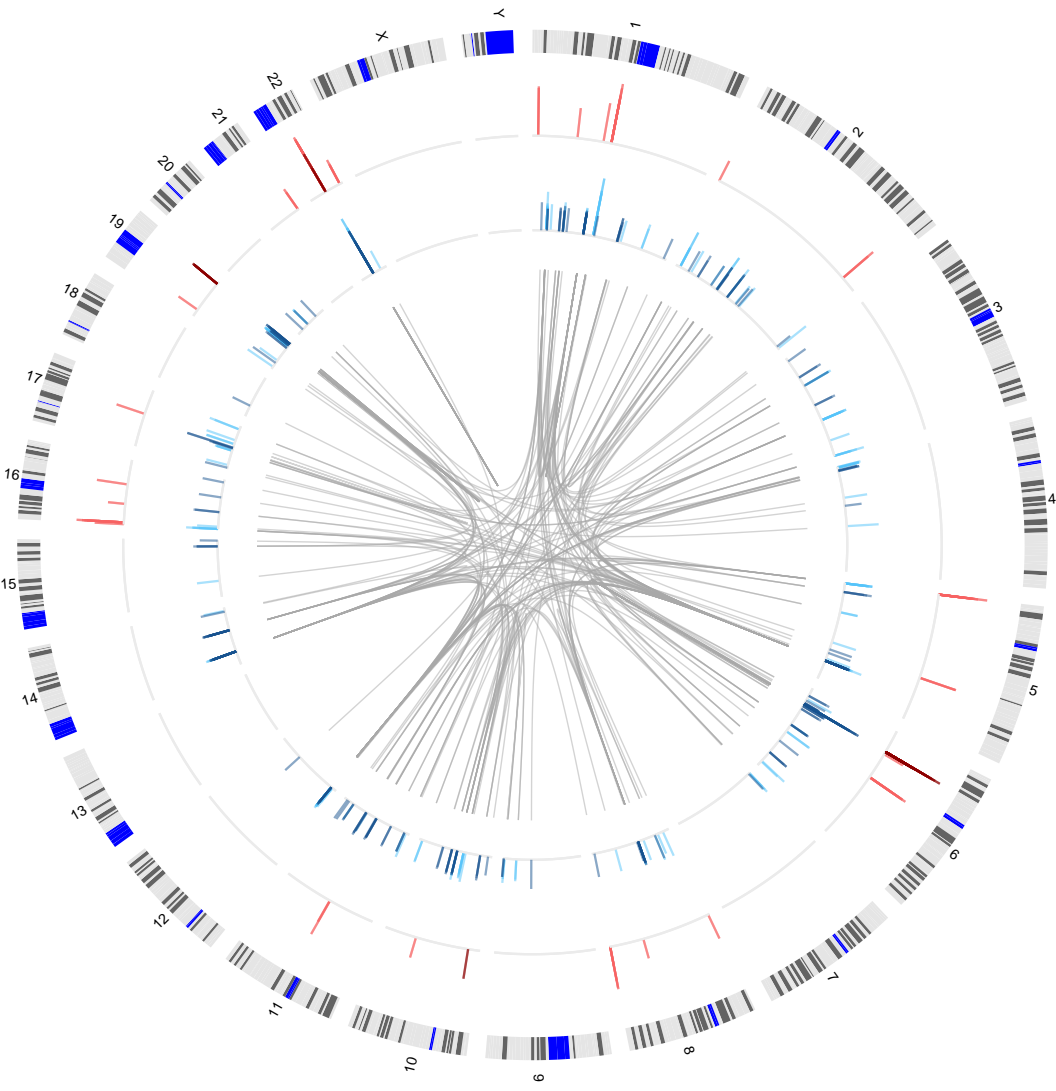

(b) PREG

Figure 2: Distribution of significant connections between DNA methylation and transcript expression across the genome in the AYATS 2a and PREG 2b) cohorts. The location of significant cis (red track) and trans relationships (blue track) across the genome (ideogram of human chromosomes, outer track) is shown. Bar graphs show the direction of the relationship (positive relationships are shown in the darker color) and the relative magnitude of the effect (height of bars; defined by adjusted R-squared values). Trans CpG-GE relationships often spanned chromosomes (location of associated CpG-GE pairs shown by center grey links). 


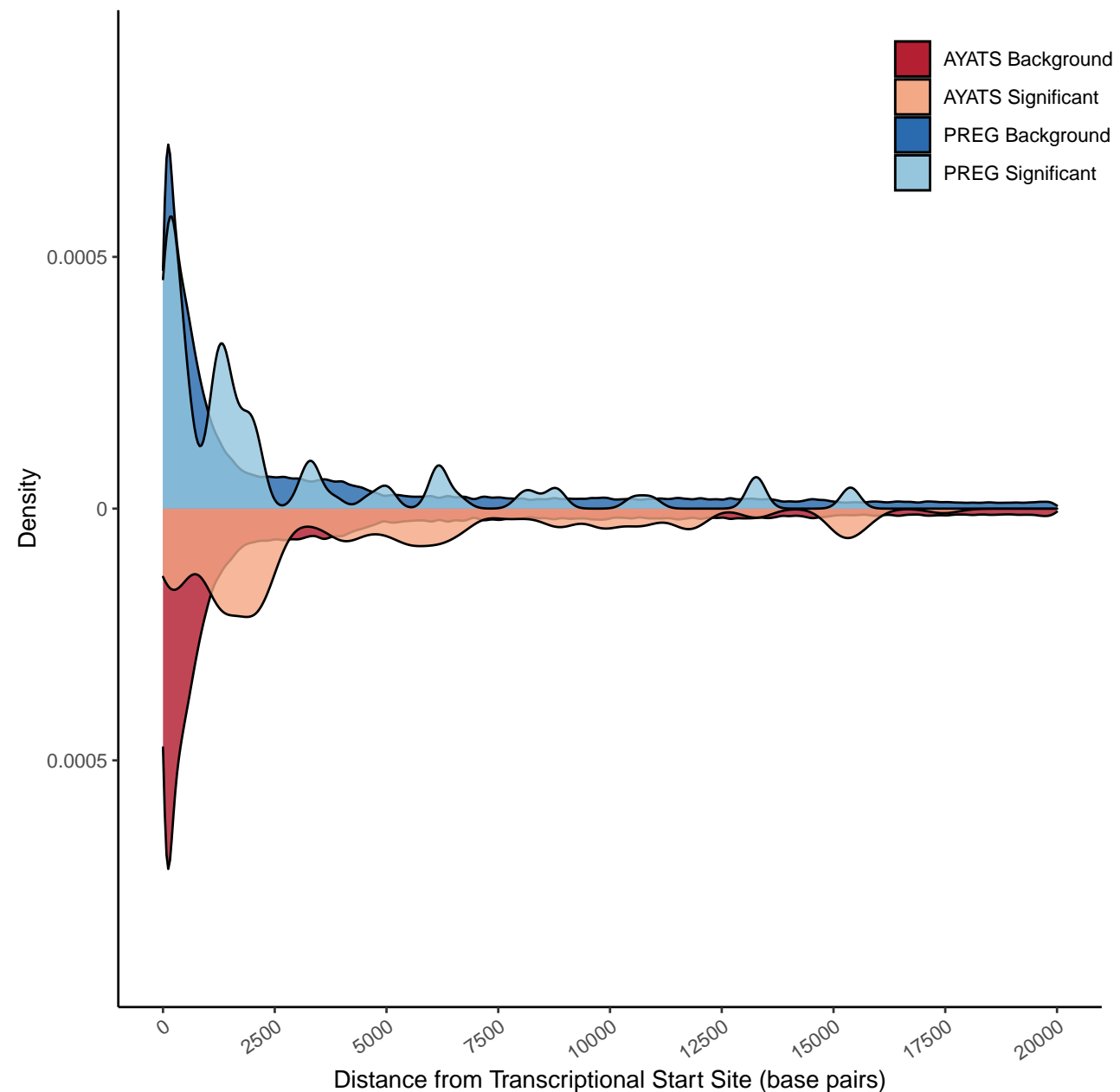

Figure 3: Absolute distance between CpG probes and transcriptional start sites (TSS) of proximal genes. A density plot depicting the relative distance of DNA methylation microarray probes (darker color) and significantly associated CpG sites (lighter color) from the transcriptional start sites of cis genes. Both the AYATS (red) and PREG (blue) cohorts showed enrichment in areas flanking active transcription. The proportion of GE-associated CpGs compared to the CpGs represented on the microarray was highest in areas directly downstream of the TSS. 
Table 3: Results of AYATS Enrichment Analyses ${ }^{1}$

\begin{tabular}{|c|c|c|c|c|}
\hline \multirow[t]{2}{*}{ Annotation } & \multicolumn{2}{|c|}{ cis } & \multicolumn{2}{|c|}{ trans } \\
\hline & enriched & depleted & enriched & depleted \\
\hline Chromatin States $^{2}$ & $\begin{array}{l}\text { TxFlnk (3), } \\
\text { ZNF/Rpts (8) } \\
\end{array}$ & $\begin{array}{l}\text { Tx (4), } \\
\text { TxWk (5), } \\
\text { Quies (15) }\end{array}$ & $\begin{array}{l}\text { TssAFlnk(2), } \\
\text { TxFlnk (3), } \\
\text { TxWk (5), } \\
\text { EnhG (6), } \\
\text { Enh (7) }\end{array}$ & $\begin{array}{l}\text { TssA (1), } \\
\text { Het (9), } \\
\text { TssBiv (10), } \\
\text { BivFlnk (11), } \\
\text { ReprPC (13), } \\
\text { ReprPCWk (14) }\end{array}$ \\
\hline CpG Classifiers & $\underline{\text { South Shore }}$ & $\begin{array}{l}\text { North shore, } \\
\text { Island }\end{array}$ & $\frac{\text { South shore }}{\text { Open sea }}$ & $\begin{array}{l}\text { North shelf, } \\
\text { North shore, } \\
\text { Island }\end{array}$ \\
\hline Gene Regions & & $\begin{array}{l}\text { 5' UTRs, } \\
\text { Promoters }\end{array}$ & Introns & $\begin{array}{l}\text { 5' UTRs, } \\
\text { Promoters }\end{array}$ \\
\hline Other $^{3}$ & $\begin{array}{l}\text { Enhancers, } \\
\text { TF binding }\end{array}$ & lncRNAs & Enhancers & $\begin{array}{l}\text { IncRNAs, } \\
\text { Insulators }\end{array}$ \\
\hline
\end{tabular}

Abbreviations. $\mathrm{UTR}=$ untranslated region, $\mathrm{TF}=$ transcription factor, $\operatorname{lncRNAs}=$ long non-coding RNAs

Bolded items. $P$-val $<0.0025$ (Bonferroni corrected for 20 tests)

Underlined items. Concordance across both the AYATS and PREG study.

$1 \frac{\text { val }<0.05}{P \text {-val }}$

${ }^{2}$ ENCODE ChromHMM 15-state model; $1=$ Active transcriptional start site (TSS), $2=$ Flanking active TSS, $3=$ Flanking strong transcription, 4= Strong transcription, 5= Weak transcription, $6=$ Genic enhancer, $7=$ Active enhancer, $8=$ Zinc-finger genes $\&$ repeats, $9=$ Heterochromatin, $10=$ Bivalent/poised TSS, 11= Flanking bivalent TSS, $12=$ Bivalent Enhancers, 13= Polycomb-repressed, 14= Weak Repressed Polycomb, 15= Quiescent

${ }^{3}$ FANTOM5-defined enhancers, transcription factor binding sites derived from ENCODE TF ChIP-seq, GENCODE long non-coding RNAs 
Table 4: Results of PREG Enrichment Analyses ${ }^{1}$

\begin{tabular}{|c|c|c|c|c|}
\hline \multirow[t]{2}{*}{ Annotation } & \multicolumn{2}{|c|}{ cis } & \multicolumn{2}{|c|}{ trans } \\
\hline & enriched & depleted & enriched & depleted \\
\hline Chromatin States ${ }^{2}$ & $\begin{array}{l}\text { TssA (1), } \\
\text { ZNF/Rpts (8) } \\
\end{array}$ & $\begin{array}{l}\text { TssAFlnk (2), } \\
\text { Tx (4) }\end{array}$ & $\begin{array}{l}\text { TssAFlnk(2), } \\
\text { TxFlnk (3), } \\
\text { Enh (7), } \\
\overline{\text { EnhBiv (12) }}\end{array}$ & $\begin{array}{l}\text { TxWk (5), } \\
\text { Het (9), } \\
\text { ReprPCWk (14), } \\
\text { Quies (15) }\end{array}$ \\
\hline CpG Classifiers & $\underline{\text { South Shore }}$ & & $\begin{array}{l}\text { North shore, } \\
\text { South shore }\end{array}$ & Island \\
\hline Gene Regions & & & Exons & $\frac{\text { Promoters, }}{3^{\prime} \text { UTRs }}$ \\
\hline Other $^{3}$ & TF binding & & $\frac{\text { Enhancers }}{\text { TF binding }}$ & \\
\hline
\end{tabular}

Abbreviations. $\mathrm{UTR}=$ untranslated region, $\mathrm{TF}=$ transcription factor, lncRNAs $=$ long noncoding RNAs

Bolded items. $P$-val $<0.0025$ (Bonferroni corrected for 20 tests)

Underlined items. Concordance across both the AYATS and PREG study.

$1 \frac{\text { Und }}{P \text {-val }<0.05}$

2 ENCODE ChromHMM 15-state model; 1= Active transcriptional start site (TSS), $2=$ Flanking active TSS, $3=$ Flanking strong transcription, $4=$ Strong transcription, 5= Weak transcription, 6= Genic enhancer, 7= Active enhancer, 8= Zinc-finger genes \& repeats, 9= Heterochromatin, 10= Bivalent/poised TSS, 11= Flanking bivalent TSS, $12=$ Bivalent Enhancers, 13= Polycomb-repressed, 14= Weak Repressed Polycomb, 15= Quiescent

${ }^{3}$ FANTOM5-defined enhancers, transcription factor binding sites derived from ENCODE TF ChIP-seq, GENCODE long non-coding RNAs 


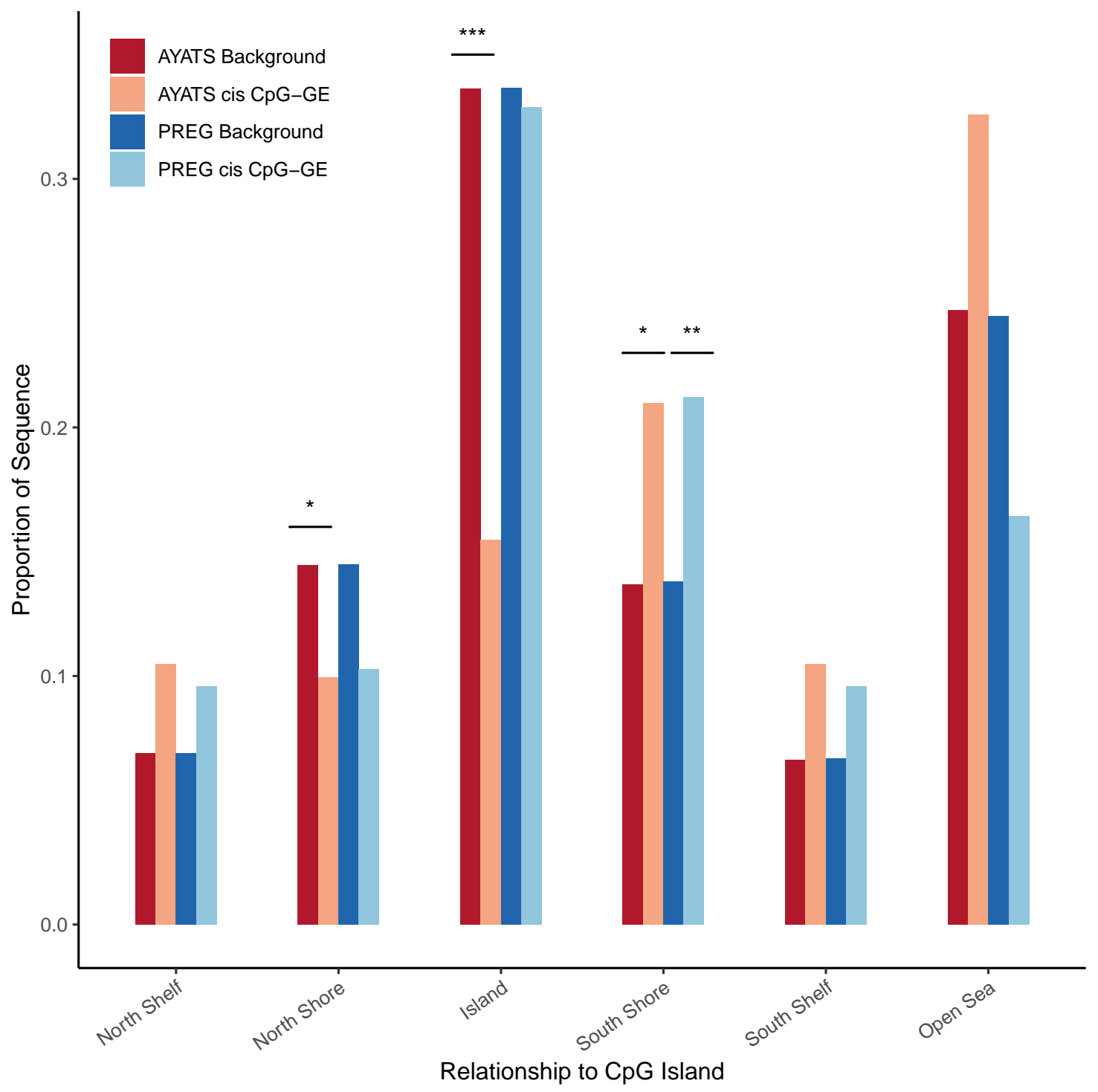

Figure 4: Enrichment for CpG classifiers in cis CpG-transcript relationships. CpG classifiers based on the distribution around $\mathrm{CpG}$ island regions were defined by the UCSC hg19 knownGene track. Islands and regions directly upstream from islands were depleted in AYATS. However, downstream regions bordering islands (South shores), were significantly enriched in both cohorts $(* * *=\mathrm{p}<0.0005 ; * *=\mathrm{p}<0.005 ; *=\mathrm{p}<0.05)$. 

available under aCC-BY-ND 4.0 International license.

A PREPRint - August 12, 2021

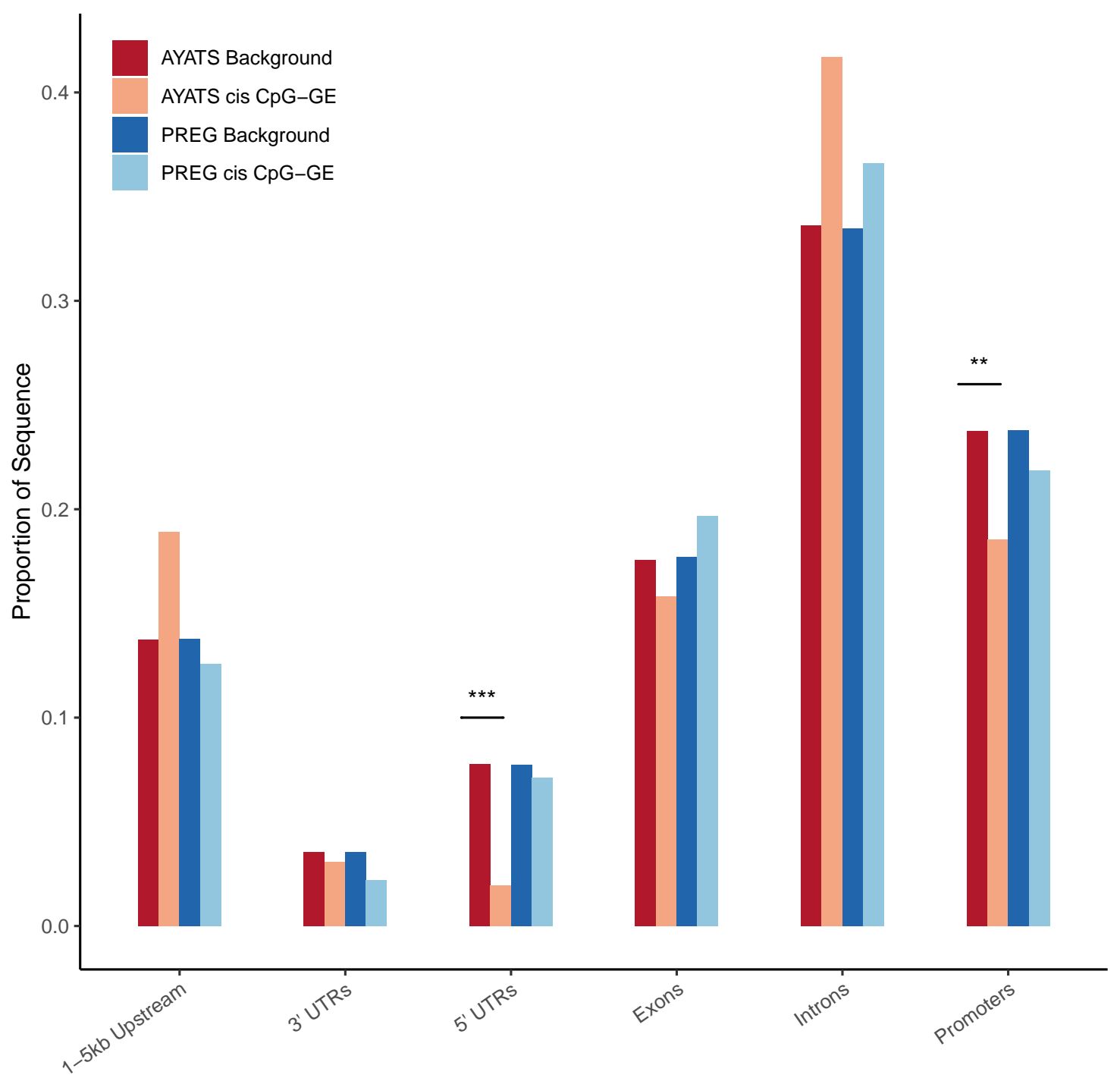

Gene Regions

Figure 5: Enrichment for gene regions in cis CpG-transcript relationships. Gene regions were annotated based on the UCSC hg19 knownGene track. GE-associated CpG sites were depleted in 5' untranslated regions (UTRs) and in promoters in the AYATS cohort only. $(* * *=\mathrm{p}<0.0005 ; * *=\mathrm{p}<0.005 ; *=\mathrm{p}<0.05)$. 


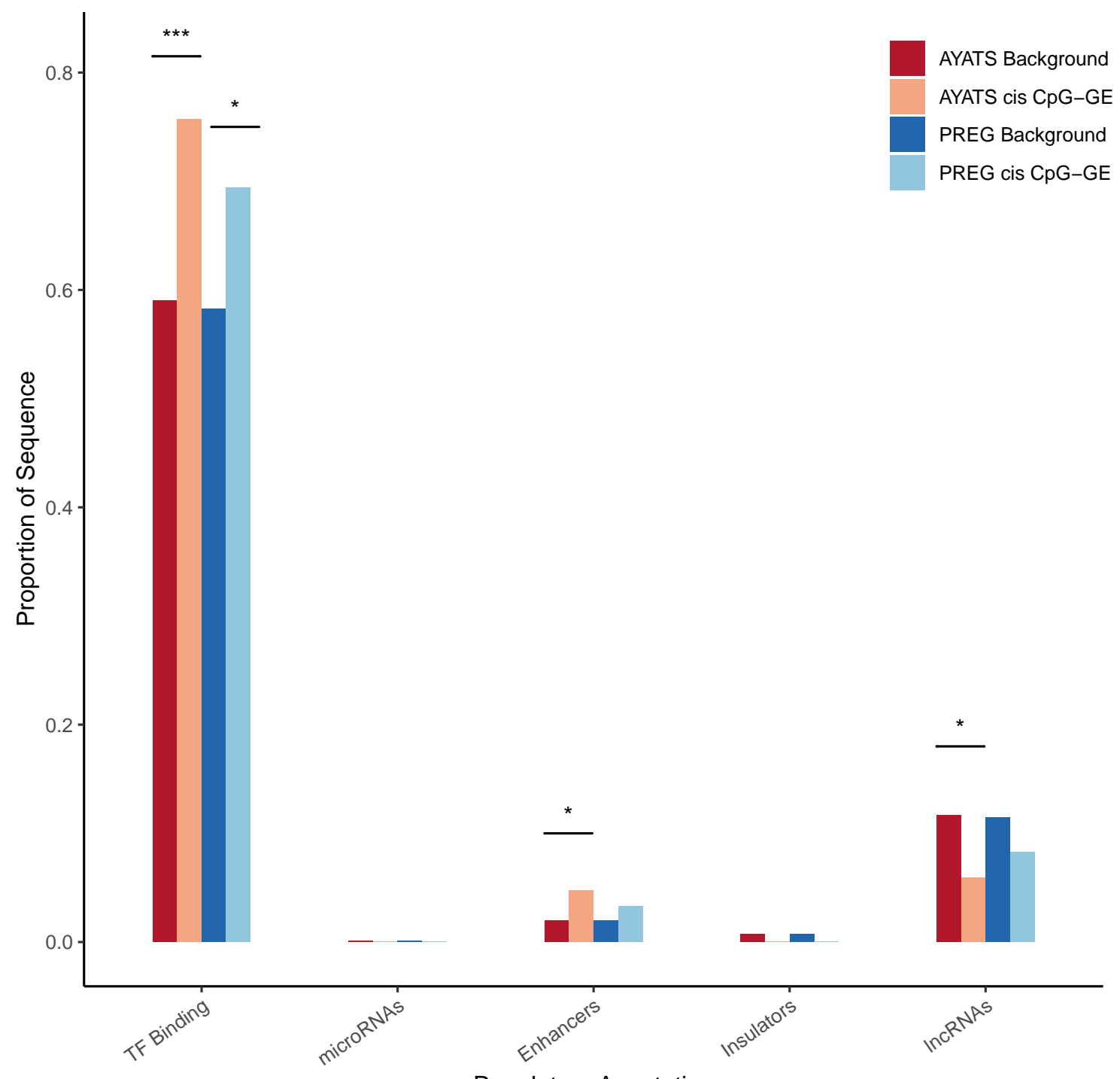

Regulatory Annotation

Figure 6: Enrichment for additional regulatory annotations in cis CpG-transcript relationships. Sites of transcription factor binding, as defined by ENCODE TF ChIP-seq annotations, were significantly enriched across cohorts. FANTOM5 enhancers were enriched in AYATS $(* * *=p<0.0005 ; * *=p<0.005 ; *=p<0.05)$. 


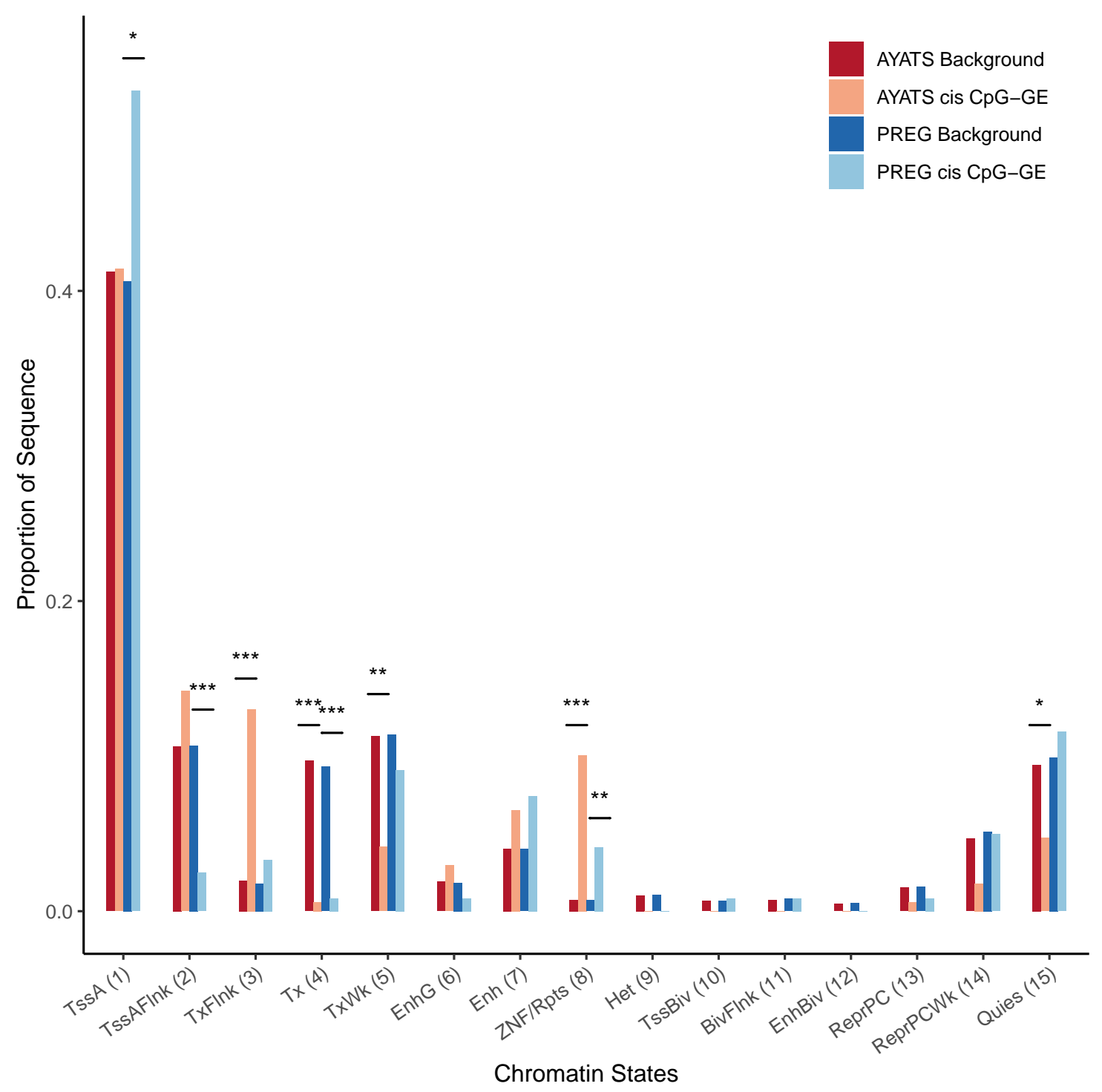

Figure 7: Enrichment for ENCODE chromatin states in cis CpG-transcript relationships. The 15-state ChromHMM model was used to determine regional chromatin states. Overall, GE-associated CpGs were depleted in transcriptionally active regions but enriched at zinc-finger binding sites $(* * *=\mathrm{p}<0.0005 ; * *=\mathrm{p}<0.005 ; *=\mathrm{p}<0.05)$. Abbreviations: 1= Active transcriptional start site (TSS), 2= Flanking active TSS, 3= Flanking strong transcription, 4= Strong transcription, 5= Weak transcription, 6= Genic enhancer, 7= Active enhancer, 8= Zinc-finger genes $\&$ repeats, 9= Heterochromatin, 10= Bivalent/poised TSS, 11= Flanking bivalent TSS, 12= Bivalent Enhancers, 13= Polycomb-repressed, 14= Weak Repressed Polycomb, 15= Quiescent 


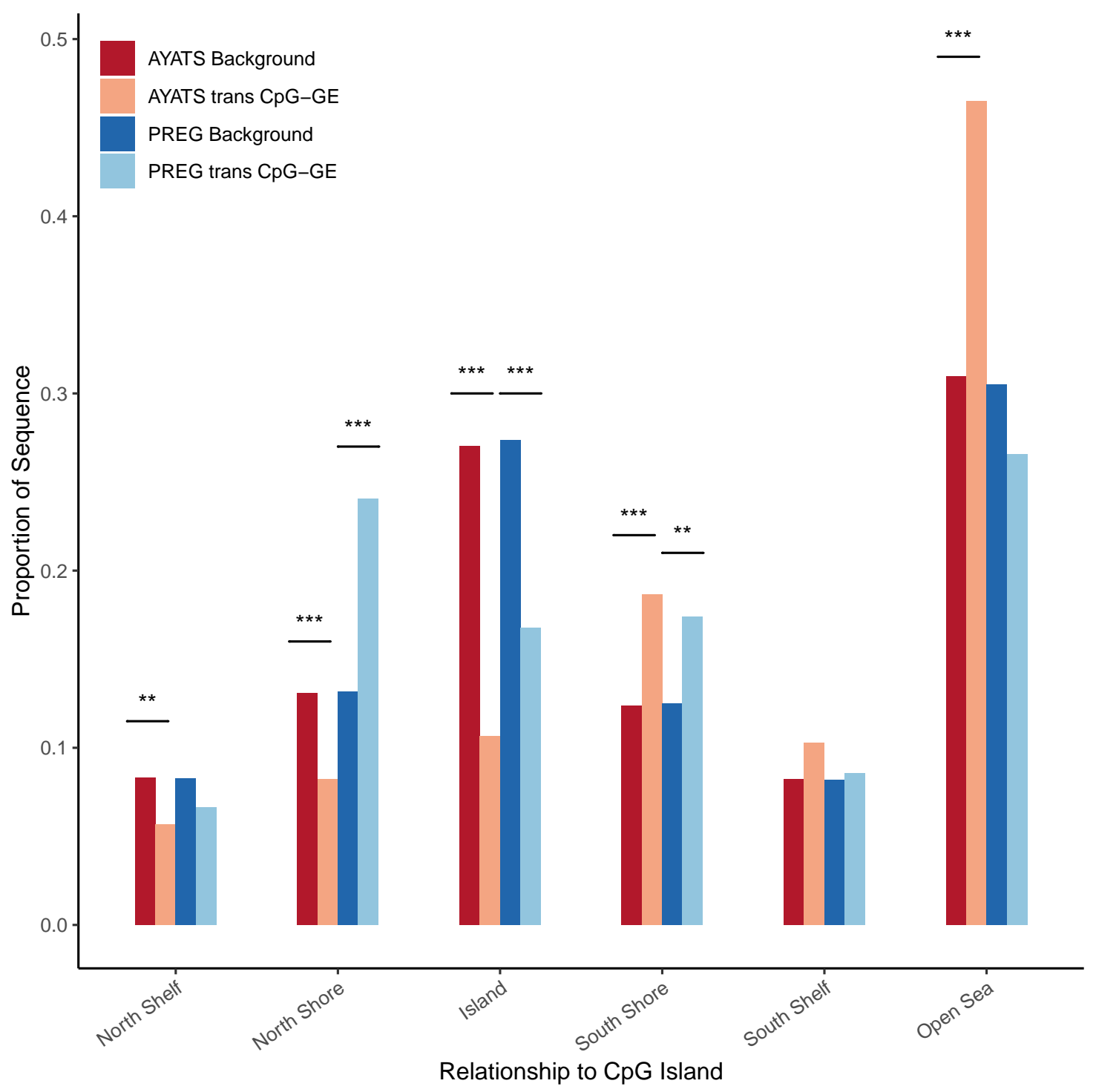

Figure 8: Enrichment for CpG classifiers in trans CpG-transcript relationships. CpG classifiers based on the distribution around $\mathrm{CpG}$ island regions were defined by the UCSC hg 19 knownGene track. Islands were depleted while downstream regions bordering islands were significantly enriched in both cohorts. The North shore region directly upstream of $\mathrm{CpG}$ islands was more variable, with significant $\mathrm{CpGs}$ showing depletion in AYATS and enrichment in PREG $(* * *=\mathrm{p}<0.0005 ; * *=\mathrm{p}<0.005 ; *=\mathrm{p}<0.05)$. 


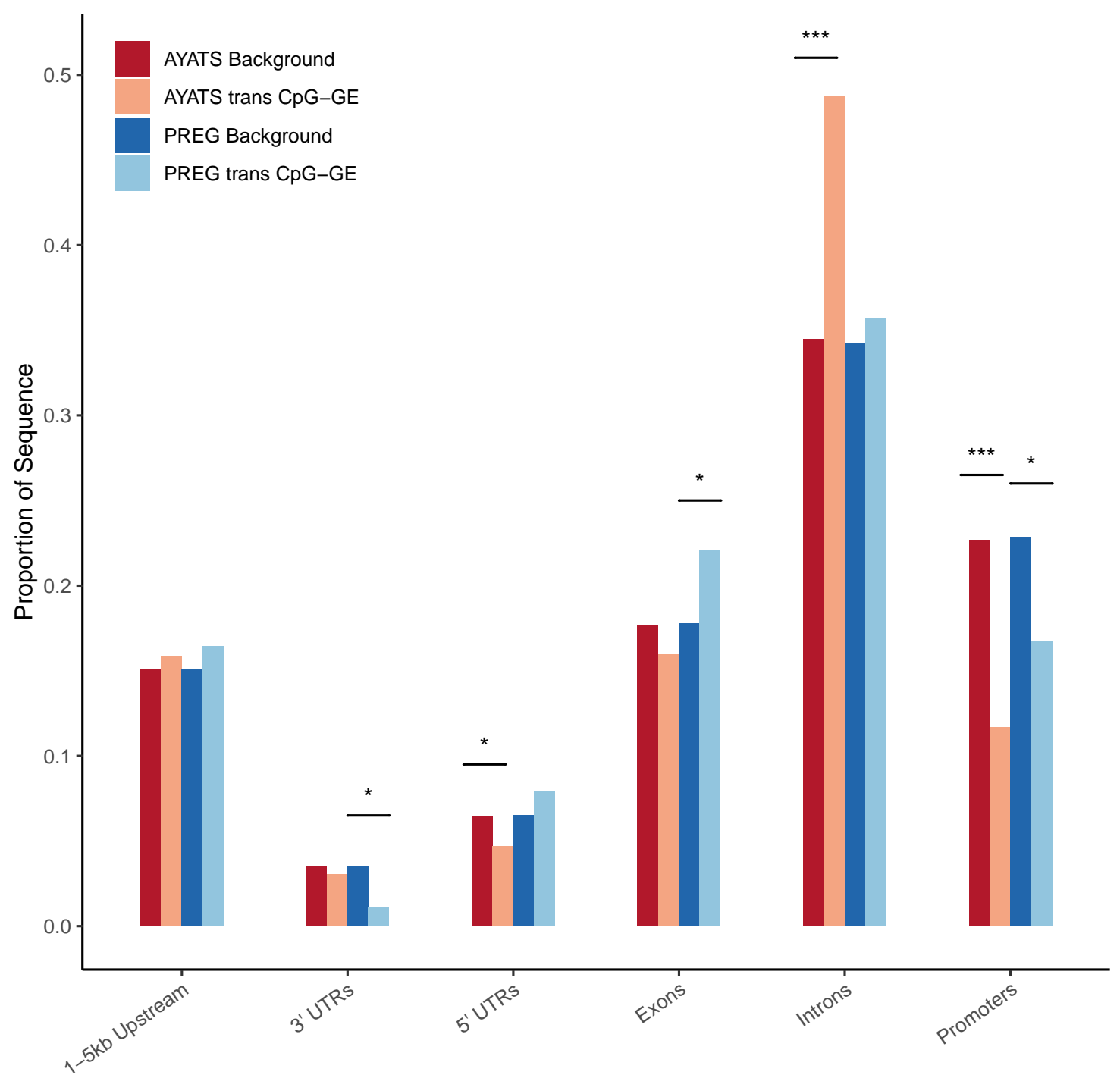

Gene Region

Figure 9: Enrichment for gene regions in trans CpG-transcript relationships. Gene regions were annotated based on the UCSC hg19 knownGene track. GE-associated CpG sites were depleted in 3' untranslated regions (PREG), 5' untranslated regions (AYATS), and in promoters (AYATS and PREG). Exons and introns were enriched in PREG and AYATS, respectively $(* * *=p<0.0005 ; * *=p<0.005 ; *=p<0.05)$. 


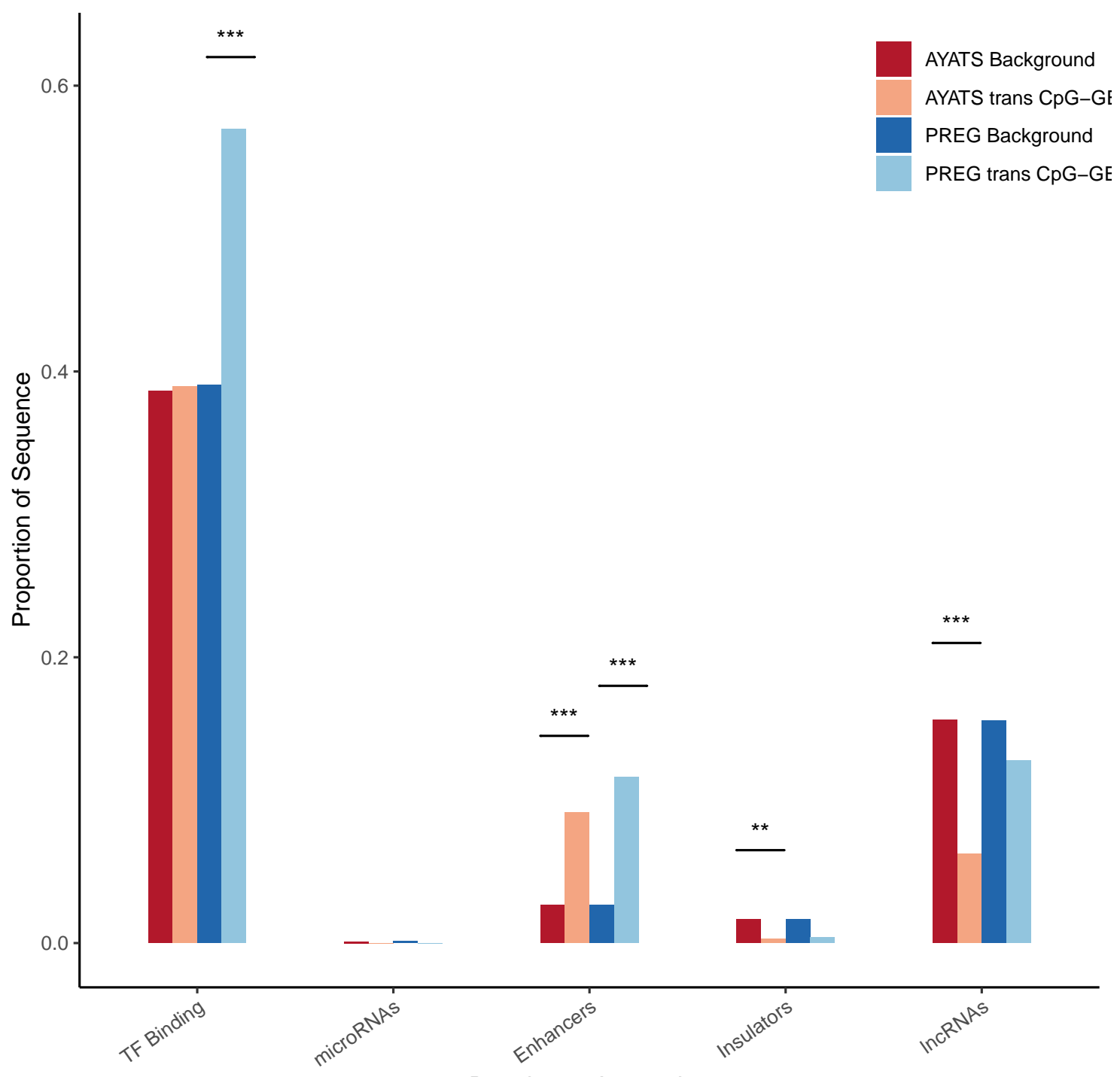

Regulatory Annotation

Figure 10: Enrichment for additional regulatory annotations in trans CpG-transcript relationships. Sites of transcription factor binding, as determined by ENCODE TF ChIP-seq, were significantly enriched in the PREG cohort. Enhancers were enriched across cohorts, and insulator regions were depleted in AYATS $(* * *=p<0.0005 ; * *=p<$ $0.005 ; *=\mathrm{p}<0.05)$. 


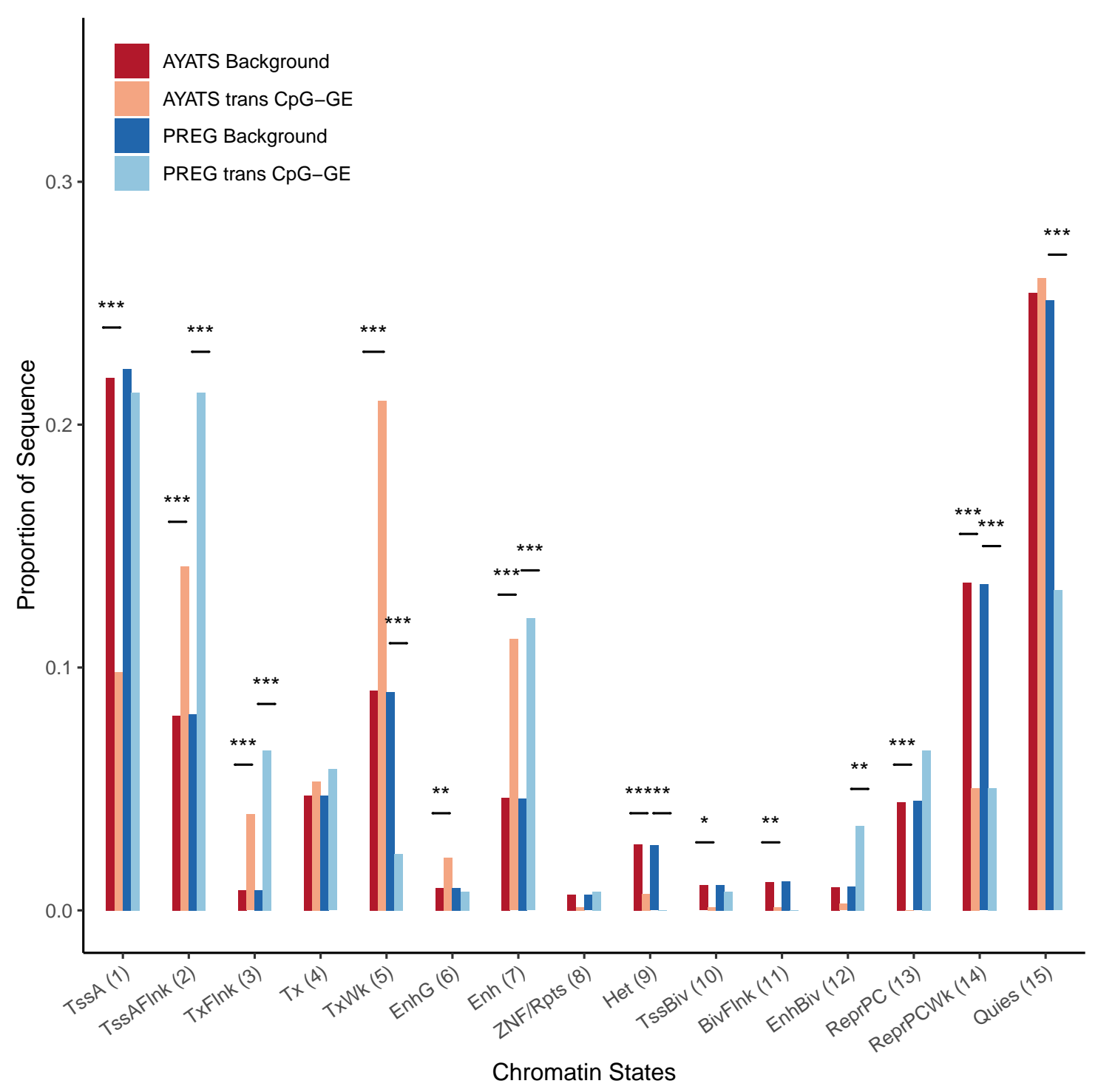

Figure 11: Enrichment for ENCODE chromatin states in trans CpG-transcript relationships. The 15-state ChromHMM model was used to determine regional chromatin states. Overall, GE-associated CpGs were depleted in repressive states but enriched at enhancers and areas flanking actively transcribed genes $(* * *=\mathrm{p}<0.0005 ; * *=\mathrm{p}<$ $0.005 ; *=\mathrm{p}<0.05)$. Abbreviations: $1=$ Active transcriptional start site (TSS), $2=$ Flanking active TSS, $3=$ Flanking strong transcription, $4=$ Strong transcription, 5= Weak transcription, $6=$ Genic enhancer, $7=$ Active enhancer, $8=$ Zinc-finger genes \& repeats, $9=$ Heterochromatin, 10= Bivalent/poised TSS, 11= Flanking bivalent TSS, 12= Bivalent Enhancers, 13= Polycomb-repressed, 14= Weak Repressed Polycomb, 15= Quiescent 
A PREPRINT - AUgust 12, 2021

\section{References}

[1] J. M. Flanagan, "Epigenome-wide association studies (EWAS): past, present, and future," Methods Mol. Biol., vol. 1238, pp. 51-63, 2015.

[2] G. Kaur, R. Begum, S. Thota, and S. Batra, "A systematic review of smoking-related epigenetic alterations," Arch. Toxicol., vol. 93, pp. 2715-2740, Oct. 2019.

[3] O. S. Anderson, K. E. Sant, and D. C. Dolinoy, "Nutrition and epigenetics: an interplay of dietary methyl donors, one-carbon metabolism and DNA methylation," J. Nutr. Biochem., vol. 23, pp. 853-859, Aug. 2012.

[4] C. H. Vinkers, A. L. Kalafateli, B. P. F. Rutten, M. J. Kas, Z. Kaminsky, J. D. Turner, and M. P. M. Boks, "Traumatic stress and human DNA methylation: a critical review," Epigenomics, vol. 7, no. 4, pp. 593-608, 2015.

[5] M. V. C. Greenberg and D. Bourc'his, "The diverse roles of DNA methylation in mammalian development and disease," Nat. Rev. Mol. Cell Biol., vol. 20, pp. 590-607, Oct. 2019.

[6] T. P. York, L. J. Eaves, M. C. Neale, and J. F. Strauss, 3rd, "The contribution of genetic and environmental factors to the duration of pregnancy," Am. J. Obstet. Gynecol., vol. 210, pp. 398-405, May 2014.

[7] R. Roberson-Nay, D. M. Lapato, A. R. Wolen, E. E. Lancaster, B. T. Webb, B. Verhulst, J. M. Hettema, and T. P. York, "An epigenome-wide association study of early-onset major depression in monozygotic twins," Transl. Psychiatry, vol. 10, p. 301, Aug. 2020.

[8] C. De Bustos, E. Ramos, J. M. Young, R. K. Tran, U. Menzel, C. F. Langford, E. E. Eichler, L. Hsu, S. Henikoff, J. P. Dumanski, and B. J. Trask, "Tissue-specific variation in DNA methylation levels along human chromosome 1.," Epigenetics Chromatin, vol. 2, p. 7, June 2009.

[9] P. A. Jones, "Functions of DNA methylation: islands, start sites, gene bodies and beyond.," Nat. Rev. Genet., vol. 13, no. 7, pp. 484-492, 2012.

[10] S. M. van der Maarel, "Epigenetic mechanisms in health and disease," Ann. Rheum. Dis., vol. 67 Suppl 3, pp. iii97-100, Dec. 2008.

[11] X. Zhang, H. J. Gierman, D. Levy, A. Plump, R. Dobrin, H. H. H. Goring, J. E. Curran, M. P. Johnson, J. Blangero, S. K. Kim, C. J. O'Donnell, V. Emilsson, and A. D. Johnson, "Synthesis of 53 tissue and cell line expression QTL datasets reveals master eQTLs," BMC Genomics, vol. 15, p. 532, June 2014.

[12] E. Hannon, O. Knox, K. Sugden, J. Burrage, C. C. Y. Wong, D. W. Belsky, D. L. Corcoran, L. Arseneault, T. E. Moffitt, A. Caspi, and J. Mill, "Characterizing genetic and environmental influences on variable DNA methylation using monozygotic and dizygotic twins," PLoS Genet., vol. 14, p. e1007544, Aug. 2018.

[13] A. L. Teh, H. Pan, L. Chen, M.-L. Ong, S. Dogra, J. Wong, J. L. MacIsaac, S. M. Mah, L. M. McEwen, S.-M. Saw, K. M. Godfrey, Y.-S. Chong, K. Kwek, C.-K. Kwoh, S.-E. Soh, M. F. F. Chong, S. Barton, N. Karnani, C. Y. Cheong, J. P. Buschdorf, W. Stünkel, M. S. Kobor, M. J. Meaney, P. D. Gluckman, and J. D. Holbrook, "The effect of genotype and in utero environment on interindividual variation in neonate DNA methylomes," Genome Res., vol. 24, pp. 1064-1074, July 2014.

[14] J. van Dongen, E. A. Ehli, R. C. Slieker, M. Bartels, Z. M. Weber, G. E. Davies, P. E. Slagboom, B. T. Heijmans, and D. I. Boomsma, "Epigenetic variation in monozygotic twins: a genome-wide analysis of DNA methylation in buccal cells," Genes, vol. 5, pp. 347-365, May 2014.

[15] T. Lappalainen and J. M. Greally, "Associating cellular epigenetic models with human phenotypes," Nat. Rev. Genet., vol. 18, pp. 441-451, July 2017.

[16] D. Schübeler, "Function and information content of DNA methylation," Nature, vol. 517, pp. 321-326, Jan. 2015.

[17] A. E. Teschendorff and C. L. Relton, "Statistical and integrative system-level analysis of DNA methylation data," Nat. Rev. Genet., vol. 19, pp. 129-147, Mar. 2018.

[18] H. Zhong, S. Kim, D. Zhi, and X. Cui, "Predicting gene expression using DNA methylation in three human populations," PeerJ, vol. 7, p. e6757, May 2019.

[19] J. Du, L. M. Johnson, S. E. Jacobsen, and D. J. Patel, "DNA methylation pathways and their crosstalk with histone methylation," Nat. Rev. Mol. Cell Biol., vol. 16, pp. 519-532, Sept. 2015.

[20] H. Liu, Y. Chen, J. Lv, H. Liu, R. Zhu, J. Su, X. Liu, Y. Zhang, and Q. Wu, "Quantitative epigenetic co-variation in CpG islands and co-regulation of developmental genes," Sci. Rep., vol. 3, p. 2576, 2013.

[21] Y. Yin, E. Morgunova, A. Jolma, E. Kaasinen, B. Sahu, S. Khund-Sayeed, P. K. Das, T. Kivioja, K. Dave, F. Zhong, K. R. Nitta, M. Taipale, A. Popov, P. A. Ginno, S. Domcke, J. Yan, D. Schübeler, C. Vinson, and J. Taipale, "Impact of cytosine methylation on DNA binding specificities of human transcription factors," Science, vol. 356, no. 6337, 2017. 
[22] A. S. Doane and O. Elemento, "Regulatory elements in molecular networks," Wiley Interdiscip. Rev. Syst. Biol. Med., vol. 9, May 2017.

[23] J. Smith, S. Sen, R. J. Weeks, M. R. Eccles, and A. Chatterjee, "Promoter DNA hypermethylation and paradoxical gene activation," Trends Cancer Res., vol. 6, pp. 392-406, May 2020.

[24] E. M. Kennedy, G. N. Goehring, M. H. Nichols, C. Robins, D. Mehta, T. Klengel, E. Eskin, A. K. Smith, and K. N. Conneely, "An integrated -omics analysis of the epigenetic landscape of gene expression in human blood cells," BMC Genomics, vol. 19, p. 476, June 2018.

[25] J. R. Wagner, S. Busche, B. Ge, T. Kwan, T. Pastinen, and M. Blanchette, "The relationship between DNA methylation, genetic and expression inter-individual variation in untransformed human fibroblasts," Genome Biol., vol. 15, p. R37, Feb. 2014.

[26] R. Lister, M. Pelizzola, R. H. Dowen, R. D. Hawkins, G. Hon, J. Tonti-Filippini, J. R. Nery, L. Lee, Z. Ye, Q.-M. Ngo, L. Edsall, J. Antosiewicz-Bourget, R. Stewart, V. Ruotti, A. H. Millar, J. A. Thomson, B. Ren, and J. R. Ecker, "Human DNA methylomes at base resolution show widespread epigenomic differences," Nature, vol. 462, pp. 315-322, Nov. 2009.

[27] D. Aran and A. Hellman, "DNA methylation of transcriptional enhancers and cancer predisposition," Cell, vol. 154, pp. 11-13, July 2013.

[28] K. E. Varley, J. Gertz, K. M. Bowling, S. L. Parker, T. E. Reddy, F. Pauli-Behn, M. K. Cross, B. A. Williams, J. A. Stamatoyannopoulos, G. E. Crawford, D. M. Absher, B. J. Wold, and R. M. Myers, "Dynamic DNA methylation across diverse human cell lines and tissues," Genome Res., vol. 23, pp. 555-567, Mar. 2013.

[29] S. Mamrut, N. Avidan, F. Truffault, E. Staun-Ram, T. Sharshar, B. Eymard, M. Frenkian, J. Pitha, M. de Baets, L. Servais, S. Berrih-Aknin, and A. Miller, "Methylome and transcriptome profiling in myasthenia gravis monozygotic twins," J. Autoimmun., vol. 82, pp. 62-73, Aug. 2017.

[30] L. Gillberg, A. Perfilyev, C. Brøns, M. Thomasen, L. G. Grunnet, P. Volkov, F. Rosqvist, D. Iggman, I. Dahlman, U. Risérus, T. Rönn, E. Nilsson, A. Vaag, and C. Ling, "Adipose tissue transcriptomics and epigenomics in low birthweight men and controls: role of high-fat overfeeding," Diabetologia, vol. 59, pp. 799-812, Apr. 2016.

[31] W. Tian, Y. Li, J. Zhang, J. Li, and J. Gao, "Combined analysis of DNA methylation and gene expression profiles of osteosarcoma identified several prognosis signatures," Gene, vol. 650, pp. 7-14, Apr. 2018.

[32] D. Song, W. Qi, M. Lv, C. Yuan, K. Tian, and F. Zhang, "Combined bioinformatics analysis reveals gene expression and DNA methylation patterns in osteoarthritis," Mol. Med. Rep., vol. 17, pp. 8069-8078, June 2018.

[33] L. Miao, R.-X. Yin, Q.-H. Zhang, X.-J. Hu, F. Huang, W.-X. Chen, X.-L. Cao, and J.-Z. Wu, "Integrated DNA methylation and gene expression analysis in the pathogenesis of coronary artery disease," Aging, vol. 11, pp. 1486-1500, Mar. 2019.

[34] Z. Wang, X. Wu, and Y. Wang, "A framework for analyzing DNA methylation data from Illumina Infinium HumanMethylation450 BeadChip," BMC Bioinformatics, vol. 19, p. 115, Apr. 2018.

[35] Y. Zhang, L. Fang, Y. Zang, and Z. Xu, "Identification of core genes and key pathways via integrated analysis of gene expression and DNA methylation profiles in bladder cancer," Med. Sci. Monit., vol. 24, pp. 3024-3033, May 2018.

[36] H. Li, F.-L. Wang, L.-P. Shan, J. An, M.-L. Liu, W. Li, J.-E. Zhang, and P.-P. Wu, "Comprehensive analysis of gene expression and DNA methylation for human nasopharyngeal carcinoma," Eur. Arch. Otorhinolaryngol., vol. 276, pp. 2565-2576, Sept. 2019.

[37] J. L. Cecilione, L. M. Rappaport, S. E. Hahn, A. E. Anderson, L. E. Hazlett, J. R. Burchett, A. A. Moore, J. E. Savage, J. M. Hettema, and R. Roberson-Nay, "Genetic and environmental contributions of negative valence systems to internalizing pathways," Twin Res. Hum. Genet., vol. 21, pp. 12-23, Feb. 2018.

[38] D. M. Lapato, S. Moyer, E. Olivares, A. B. Amstadter, P. A. Kinser, S. J. Latendresse, C. Jackson-Cook, R. Roberson-Nay, J. F. Strauss, and T. P. York, "Prospective longitudinal study of the pregnancy DNA methylome: the US Pregnancy, Race, Environment, Genes (PREG) study.," BMJ Open, vol. 8, p. e019721, May 2018.

[39] R Core Team, "R: A language and environment for statistical computing. R Foundation for Statistical Computing, Vienna, Austria.," 2016.

[40] E. A. Houseman, W. P. Accomando, D. C. Koestler, B. C. Christensen, C. J. Marsit, H. H. Nelson, J. K. Wiencke, and K. T. Kelsey, "DNA methylation arrays as surrogate measures of cell mixture distribution.," $B M C$ Bioinformatics, vol. 13, p. 86, May 2012. 
[41] M. Maes, H. Y. Meltzer, W. Stevens, J. Calabrese, and P. Cosyns, "Natural killer cell activity in major depression: relation to circulating natural killer cells, cellular indices of the immune response, and depressive phenomenology," Prog. Neuropsychopharmacol. Biol. Psychiatry, vol. 18, pp. 717-730, July 1994.

[42] R. T. Barfield, L. M. Almli, V. Kilaru, A. K. Smith, K. B. Mercer, R. Duncan, T. Klengel, D. Mehta, E. B. Binder, M. P. Epstein, K. J. Ressler, and K. N. Conneely, "Accounting for population stratification in DNA methylation studies," Genet. Epidemiol., vol. 38, pp. 231-241, Apr. 2014.

[43] M. E. Ritchie, B. Phipson, D. Wu, Y. Hu, C. W. Law, W. Shi, and G. K. Smyth, "limma powers differential expression analyses for RNA-sequencing and microarray studies," Nucleic Acids Research, vol. 43, no. 7, p. e47, 2015.

[44] J. Guintivano, A. A. Shabalin, R. F. Chan, D. R. Rubinow, P. F. Sullivan, S. Meltzer-Brody, K. A. Aberg, and E. J. C. G. van den Oord, "Test-statistic inflation in methylome-wide association studies," Epigenetics, pp. 1-4, May 2020.

[45] J. R. Goñi, A. Pérez, D. Torrents, and M. Orozco, "Determining promoter location based on DNA structure first-principles calculations," Genome Biol., vol. 8, no. 12, p. R263, 2007.

[46] M. Brandeis, D. Frank, I. Keshet, Z. Siegfried, M. Mendelsohn, A. Nemes, V. Temper, A. Razin, and H. Cedar, "Sp1 elements protect a CpG island from de novo methylation," Nature, vol. 371, pp. 435-438, Sept. 1994.

[47] P. Mummaneni, P. Yates, J. Simpson, J. Rose, and M. S. Turker, "The primary function of a redundant sp1 binding site in the mouse aprt gene promoter is to block epigenetic gene inactivation," Nucleic Acids Res., vol. 26, pp. 5163-5169, Nov. 1998.

[48] A. Feldmann, R. Ivanek, R. Murr, D. Gaidatzis, L. Burger, and D. Schübeler, "Transcription factor occupancy can mediate active turnover of DNA methylation at regulatory regions," PLoS Genet., vol. 9, p. e1003994, Dec. 2013.

[49] F.-F. Xie, F.-Y. Deng, L.-F. Wu, X.-B. Mo, H. Zhu, J. Wu, Y.-F. Guo, K.-Q. Zeng, M.-J. Wang, X.-W. Zhu, W. Xia, L. Wang, P. He, P.-F. Bing, X. Lu, Y.-H. Zhang, and S.-F. Lei, "Multiple correlation analyses revealed complex relationship between DNA methylation and mRNA expression in human peripheral blood mononuclear cells," Funct. Integr. Genomics, vol. 18, pp. 1-10, Jan. 2018.

[50] Y. Zhao, H. Sun, and H. Wang, "Long noncoding RNAs in DNA methylation: new players stepping into the old game," Cell Biosci., vol. 6, p. 45, July 2016.

[51] L. Yu, K. Xia, X. Cen, X. Huang, W. Sun, Z. Zhao, and J. Liu, "DNA methylation of noncoding RNAs: new insights into osteogenesis and common bone diseases," Stem Cell Res. Ther., vol. 11, p. 109, Mar. 2020.

[52] Roadmap Epigenomics Consortium, A. Kundaje, W. Meuleman, J. Ernst, M. Bilenky, A. Yen, A. Heravi-Moussavi, P. Kheradpour, Z. Zhang, J. Wang, M. J. Ziller, V. Amin, J. W. Whitaker, M. D. Schultz, L. D. Ward, A. Sarkar, G. Quon, R. S. Sandstrom, M. L. Eaton, Y.-C. Wu, A. R. Pfenning, X. Wang, M. Claussnitzer, Y. Liu, C. Coarfa, R. A. Harris, N. Shoresh, C. B. Epstein, E. Gjoneska, D. Leung, W. Xie, R. D. Hawkins, R. Lister, C. Hong, P. Gascard, A. J. Mungall, R. Moore, E. Chuah, A. Tam, T. K. Canfield, R. S. Hansen, R. Kaul, P. J. Sabo, M. S. Bansal, A. Carles, J. R. Dixon, K.-H. Farh, S. Feizi, R. Karlic, A.-R. Kim, A. Kulkarni, D. Li, R. Lowdon, G. Elliott, T. R. Mercer, S. J. Neph, V. Onuchic, P. Polak, N. Rajagopal, P. Ray, R. C. Sallari, K. T. Siebenthall, N. A. Sinnott-Armstrong, M. Stevens, R. E. Thurman, J. Wu, B. Zhang, X. Zhou, A. E. Beaudet, L. A. Boyer, P. L. De Jager, P. J. Farnham, S. J. Fisher, D. Haussler, S. J. M. Jones, W. Li, M. A. Marra, M. T. McManus, S. Sunyaev, J. A. Thomson, T. D. Tlsty, L.-H. Tsai, W. Wang, R. A. Waterland, M. Q. Zhang, L. H. Chadwick, B. E. Bernstein, J. F. Costello, J. R. Ecker, M. Hirst, A. Meissner, A. Milosavljevic, B. Ren, J. A. Stamatoyannopoulos, T. Wang, and M. Kellis, "Integrative analysis of 111 reference human epigenomes," Nature, vol. 518, pp. 317-330, Feb. 2015.

[53] J. Ernst and M. Kellis, "ChromHMM: automating chromatin-state discovery and characterization," Nat. Methods, vol. 9, pp. 215-216, Feb. 2012.

[54] M. Gardiner-Garden and M. Frommer, "CpG islands in vertebrate genomes," J. Mol. Biol., vol. 196, pp. 261-282, July 1987.

[55] M. E. Price, A. M. Cotton, L. L. Lam, P. Farré, E. Emberly, C. J. Brown, W. P. Robinson, and M. S. Kobor, "Additional annotation enhances potential for biologically-relevant analysis of the Illumina Infinium HumanMethylation450 BeadChip array," Epigenetics Chromatin, vol. 6, p. 4, Mar. 2013.

[56] ENCODE Project Consortium, "An integrated encyclopedia of DNA elements in the human genome," Nature, vol. 489, pp. 57-74, Sept. 2012.

[57] J. Harrow, A. Frankish, J. M. Gonzalez, E. Tapanari, M. Diekhans, F. Kokocinski, B. L. Aken, D. Barrell, A. Zadissa, S. Searle, I. Barnes, A. Bignell, V. Boychenko, T. Hunt, M. Kay, G. Mukherjee, J. Rajan, G. DespacioReyes, G. Saunders, C. Steward, R. Harte, M. Lin, C. Howald, A. Tanzer, T. Derrien, J. Chrast, N. Walters, 
S. Balasubramanian, B. Pei, M. Tress, J. M. Rodriguez, I. Ezkurdia, J. van Baren, M. Brent, D. Haussler, M. Kellis, A. Valencia, A. Reymond, M. Gerstein, R. Guigó, and T. J. Hubbard, "GENCODE: the reference human genome annotation for the ENCODE project," Genome Res., vol. 22, pp. 1760-1774, Sept. 2012.

[58] R. Andersson, C. Gebhard, I. Miguel-Escalada, I. Hoof, J. Bornholdt, M. Boyd, Y. Chen, X. Zhao, C. Schmidl, T. Suzuki, E. Ntini, E. Arner, E. Valen, K. Li, L. Schwarzfischer, D. Glatz, J. Raithel, B. Lilje, N. Rapin, F. O. Bagger, M. Jørgensen, P. R. Andersen, N. Bertin, O. Rackham, A. M. Burroughs, J. K. Baillie, Y. Ishizu, Y. Shimizu, E. Furuhata, S. Maeda, Y. Negishi, C. J. Mungall, T. F. Meehan, T. Lassmann, M. Itoh, H. Kawaji, N. Kondo, J. Kawai, A. Lennartsson, C. O. Daub, P. Heutink, D. A. Hume, T. H. Jensen, H. Suzuki, Y. Hayashizaki, F. Müller, A. R. R. Forrest, P. Carninci, M. Rehli, and A. Sandelin, "An atlas of active enhancers across human cell types and tissues," Nature, vol. 507, pp. 455-461, Mar. 2014.

[59] D. Anastasiadi, A. Esteve-Codina, and F. Piferrer, "Consistent inverse correlation between DNA methylation of the first intron and gene expression across tissues and species," Epigenetics Chromatin, vol. 11, p. 37, June 2018.

[60] M. Carlson and B. P. Maintainer, TxDb.Hsapiens.UCSC.hg19.knownGene: Annotation package for TxDb object(s), 2015. R package version 3.2.2.

[61] Y. Benjamini and Y. Hochberg, "Controlling the false discovery rate: A practical and powerful approach to multiple testing," J. R. Stat. Soc. Series B Stat. Methodol., vol. 57, no. 1, pp. 289-300, 1995.

[62] J. Cheng, D. Wei, Y. Ji, L. Chen, L. Yang, G. Li, L. Wu, T. Hou, L. Xie, G. Ding, H. Li, and Y. Li, "Integrative analysis of DNA methylation and gene expression reveals hepatocellular carcinoma-specific diagnostic biomarkers," Genome Med., vol. 10, p. 42, May 2018.

[63] H. Zhu, G. Wang, and J. Qian, "Transcription factors as readers and effectors of DNA methylation," Nat. Rev. Genet., vol. 17, pp. 551-565, Aug. 2016.

[64] A. K. Maunakea, R. P. Nagarajan, M. Bilenky, T. J. Ballinger, C. D’Souza, S. D. Fouse, B. E. Johnson, C. Hong, C. Nielsen, Y. Zhao, G. Turecki, A. Delaney, R. Varhol, N. Thiessen, K. Shchors, V. M. Heine, D. H. Rowitch, X. Xing, C. Fiore, M. Schillebeeckx, S. J. M. Jones, D. Haussler, M. A. Marra, M. Hirst, T. Wang, and J. F. Costello, "Conserved role of intragenic DNA methylation in regulating alternative promoters," Nature, vol. 466, pp. 253-257, July 2010.

[65] G. Lev Maor, A. Yearim, and G. Ast, “The alternative role of DNA methylation in splicing regulation," Trends Genet., vol. 31, pp. 274-280, May 2015.

[66] R. A. Irizarry, C. Ladd-Acosta, B. Wen, Z. Wu, C. Montano, P. Onyango, H. Cui, K. Gabo, M. Rongione, M. Webster, H. Ji, J. Potash, S. Sabunciyan, and A. P. Feinberg, "The human colon cancer methylome shows similar hypo- and hypermethylation at conserved tissue-specific CpG island shores," Nat. Genet., vol. 41, pp. 178186, Feb. 2009.

[67] R. K. Slotkin and R. Martienssen, "Transposable elements and the epigenetic regulation of the genome," Nat. Rev. Genet., vol. 8, pp. 272-285, Apr. 2007.

[68] Y. Liu, J. Ding, L. M. Reynolds, K. Lohman, T. C. Register, A. De La Fuente, T. D. Howard, G. A. Hawkins, W. Cui, J. Morris, S. G. Smith, R. G. Barr, J. D. Kaufman, G. L. Burke, W. Post, S. Shea, C. E. McCall, D. Siscovick, D. R. Jacobs, Jr, R. P. Tracy, D. M. Herrington, and I. Hoeschele, "Methylomics of gene expression in human monocytes," Hum. Mol. Genet., vol. 22, pp. 5065-5074, Dec. 2013.

[69] A. Doi, I.-H. Park, B. Wen, P. Murakami, M. J. Aryee, R. Irizarry, B. Herb, C. Ladd-Acosta, J. Rho, S. Loewer, J. Miller, T. Schlaeger, G. Q. Daley, and A. P. Feinberg, "Differential methylation of tissue- and cancer-specific CpG island shores distinguishes human induced pluripotent stem cells, embryonic stem cells and fibroblasts," Nat. Genet., vol. 41, pp. 1350-1353, Dec. 2009.

[70] N. D. Vanderkraats, J. F. Hiken, K. F. Decker, and J. R. Edwards, "Discovering high-resolution patterns of differential DNA methylation that correlate with gene expression changes," Nucleic Acids Res., vol. 41, pp. 68166827, Aug. 2013.

[71] C. E. Schlosberg, N. D. VanderKraats, and J. R. Edwards, "Modeling complex patterns of differential DNA methylation that associate with gene expression changes," Nucleic Acids Res., vol. 45, pp. 5100-5111, May 2017.

[72] M. Bulger and M. Groudine, "Functional and mechanistic diversity of distal transcription enhancers," Cell, vol. 144, pp. 327-339, Feb. 2011.

[73] S. Schoenfelder and P. Fraser, "Long-range enhancer-promoter contacts in gene expression control," Nat. Rev. Genet., vol. 20, pp. 437-455, Aug. 2019.

[74] B. H. Chen, R. E. Marioni, E. Colicino, M. J. Peters, C. K. Ward-Caviness, P.-C. Tsai, N. S. Roetker, A. C. Just, E. W. Demerath, W. Guan, J. Bressler, M. Fornage, S. Studenski, A. R. Vandiver, A. Z. Moore, T. Tanaka, D. P. 
Kiel, L. Liang, P. Vokonas, J. Schwartz, K. L. Lunetta, J. M. Murabito, S. Bandinelli, D. G. Hernandez, D. Melzer, M. Nalls, L. C. Pilling, T. R. Price, A. B. Singleton, C. Gieger, R. Holle, A. Kretschmer, F. Kronenberg, S. Kunze, J. Linseisen, C. Meisinger, W. Rathmann, M. Waldenberger, P. M. Visscher, S. Shah, N. R. Wray, A. F. McRae, O. H. Franco, A. Hofman, A. G. Uitterlinden, D. Absher, T. Assimes, M. E. Levine, A. T. Lu, P. S. Tsao, L. Hou, J. E. Manson, C. L. Carty, A. Z. LaCroix, A. P. Reiner, T. D. Spector, A. P. Feinberg, D. Levy, A. Baccarelli, J. van Meurs, J. T. Bell, A. Peters, I. J. Deary, J. S. Pankow, L. Ferrucci, and S. Horvath, "DNA methylation-based measures of biological age: meta-analysis predicting time to death.," Aging, vol. 8, pp. 1844-1865, Sept. 2016.

[75] F. R. Pinu, D. J. Beale, A. M. Paten, K. Kouremenos, S. Swarup, H. J. Schirra, and D. Wishart, "Systems biology and multi-omics integration: Viewpoints from the metabolomics research community," Metabolites, vol. 9, Apr. 2019.

[76] R. Haas, A. Zelezniak, J. Iacovacci, S. Kamrad, S. Townsend, and M. Ralser, "Designing and interpreting 'multiomic' experiments that may change our understanding of biology," Current Opinion in Systems Biology, vol. 6, pp. 37-45, Dec. 2017.

[77] I. Subramanian, S. Verma, S. Kumar, A. Jere, and K. Anamika, "Multi-omics data integration, interpretation, and its application,” Bioinform. Biol. Insights, vol. 14, p. 1177932219899051, Jan. 2020.

[78] M. Bersanelli, E. Mosca, D. Remondini, E. Giampieri, C. Sala, G. Castellani, and L. Milanesi, "Methods for the integration of multi-omics data: mathematical aspects," BMC Bioinformatics, vol. 17 Suppl 2, p. 15, Jan. 2016.

[79] K. R. van Eijk, S. de Jong, M. P. M. Boks, T. Langeveld, F. Colas, J. H. Veldink, C. G. F. de Kovel, E. Janson, E. Strengman, P. Langfelder, R. S. Kahn, L. H. van den Berg, S. Horvath, and R. A. Ophoff, "Genetic analysis of DNA methylation and gene expression levels in whole blood of healthy human subjects," BMC Genomics, vol. 13, p. 636, Nov. 2012.

[80] D. L. Taylor, A. U. Jackson, N. Narisu, G. Hemani, M. R. Erdos, P. S. Chines, A. Swift, J. Idol, J. P. Didion, R. P. Welch, L. Kinnunen, J. Saramies, T. A. Lakka, M. Laakso, J. Tuomilehto, S. C. J. Parker, H. A. Koistinen, G. Davey Smith, M. Boehnke, L. J. Scott, E. Birney, and F. S. Collins, "Integrative analysis of gene expression, DNA methylation, physiological traits, and genetic variation in human skeletal muscle," Proc. Natl. Acad. Sci. U. S. A., vol. 116, pp. 10883-10888, May 2019.

[81] M. Bibikova, B. Barnes, C. Tsan, V. Ho, B. Klotzle, J. M. Le, D. Delano, L. Zhang, G. P. Schroth, K. L. Gunderson, J.-B. Fan, and R. Shen, "High density DNA methylation array with single CpG site resolution," Genomics, vol. 98, pp. 288-295, Oct. 2011.

[82] E. Birney, G. D. Smith, and J. M. Greally, "Epigenome-wide association studies and the interpretation of disease -omics," PLoS Genet., vol. 12, p. e1006105, June 2016.

[83] A. Pacis, F. Mailhot-Léonard, L. Tailleux, H. E. Randolph, V. Yotova, A. Dumaine, J.-C. Grenier, and L. B. Barreiro, "Gene activation precedes DNA demethylation in response to infection in human dendritic cells," Proc. Natl. Acad. Sci. U. S. A., vol. 116, pp. 6938-6943, Apr. 2019.

[84] L. Sie, S. Loong, and E. K. Tan, “Utility of lymphoblastoid cell lines,” J. Neurosci. Res., vol. 87, pp. 1953-1959, July 2009.

[85] W.-S. Yong, F.-M. Hsu, and P.-Y. Chen, "Profiling genome-wide DNA methylation," Epigenetics Chromatin, vol. 9, p. 26, June 2016. 